\title{
Self-organization in semiconductor lasers with ultra-short optical feedback
}

\author{
DISSERTATION \\ zur Erlangung des akademischen Grades \\ doctor rerum naturalium \\ (Dr. rer. nat.) \\ im Fach Physik \\ eingereicht an der \\ Mathematisch-Naturwissenschaftlichen Fakultät I \\ Humboldt-Universität zu Berlin \\ von \\ Herr Dipl.-Phys. Oleg Ushakov \\ geboren am 01.03.1978 in Kislowodsk, Russland
}

Präsident der Humboldt-Universität zu Berlin:

Prof. Dr. Christoph Markschies

Dekan der Mathematisch-Naturwissenschaftlichen Fakultät I:

Prof. Dr. Cristian Limberg

Gutachter:

1. Prof. Dr.- Fritz Henneberger

2. Prof. Dr.- Lutz Schimansky-Geier

3. Prof. Dr.-Wolfgang Elsäßer

eingereicht am:

8. Januar 2007

Tag der mündlichen Prüfung: 23. April 2007 


\begin{abstract}
In this work, self-organization in semiconductor lasers with ultra-short optical feedback is investigated. Exploiting dc currents to tune the relevant feedback parameters, we have experimentally prepared and studied a number of novel nonlinear dynamical scenarios.

Two different types of self-sustaining intensity-pulsations are detected depending on strength and phase of the feedback. One type of pulsations is emerging in a Hopf-bifurcation from relaxation oscillations. These oscillations become undamped due to dispersive self-Q switching. The second type of pulsations is a beating of distinct compound-cavity modes. It is also born in a Hopf bifurcation. These findings represent experimental evidence for theoretical predictions. A supplementary mode and stability analysis agrees well with measurements.

Coexistence of mode beating and relaxation oscillations gives rise to the break-up of regular pulsations into chaotic emission via a quasi-periodic route to chaos. The sudden destruction of chaos is indicative of a boundary crisis scenario, in which we see a discontinuous disappearance of an attractor. The existence of chaotic saddles underlying transient chaotic dynamics which appears behind boundary crisis is experimentally verified. It is experimentally demonstrated that an excitation of chaotic transients is closely related to a conventional excitability. The experiment is supplemented by numerical simulations.

The influence of external Gaussian noise close to the onset of sub- and super-critical Hopf bifurcations is studied. Noise-induced oscillations appear as a noisy precursor with Lorentzian shape peak in the power spectrum. The coherence factor defined by the product of height and quality factor exhibits non-monotonic behavior with a distinct maximum at a certain noise intensity for both types of Hopf bifurcations, demonstrating coherence resonance. Besides these similarities, the measurements reveal also qualitative differences between the two cases. Whereas the width of the noise induced peak increases monotonically with noise intensity for the supercritical bifurcation, it traverses a pronounced minimum in the subcritical case. The experimental findings are examined in terms of general model for the noise driven motion close to bifurcations.
\end{abstract}

\title{
Keywords:
}

Nonlinear dynamics, Coherence resonance, Excitability, Chaos 


\section{Zusammenfassung}

In dieser Arbeit wird die Selbstorganisation in Halbleiterlasern mit ultrakurzer optischer Rückkopplung untersucht. Es wurden eine Vielzahl neuer nichtlinearer dynamischer Szenarien experimentell präpariert und untersucht, wobei die Steuerung der relevanten Rückkopplungsparameter über Injektionsströme erfolgt.

Zwei verschiedene Typen von selbsterhaltenden Intensitätspulsationen wurden abhängig von der Phase und der Stärke der Rückkopplung gefunden. Ein Pulsationstyp entsteht in einer Hopf-Bifurkation aus gedämpften Relaxationsoszillationen. Diese Oszillationen werden durch dispersives Selbst-QSchalten entdämpft. Beim zweiten Pulsationstyp handelt es sich um Schwebungs Oszillationen zweier verschiedener konkurrierender Moden der Gesamtkavität. Sie werden ebenfalls in einer Hopf-Bifurkation geboren. Diese Ergebnisse repräsentieren experimentelle Beweise für theoretische Vorhersagen. Eine zusätzlich durchgeführte Moden- und Stabilitätsanalyse zeigt gute übereinstimmung mit den Messergebnissen.

Die Koexistenz von Schwebungsoszillationen und Relaxationsoszillationen führt zum übergang von regulären Pulsationen in chaotische Emission über eine quasiperiodische Route zum Chaos. Ein plötzlicher Untergang des Chaos deutet auf ein Randbedingungs-Krisen-Szenario hin, wo wir ein unstetiges Verschwinden eines Attraktors beobachten. Die Existenz chaotischer Sättel, die transienten chaotischen Dynamiken nach einer Randbedingungskrise zugrunde liegen, wird experimentell verifiziert. Es wird experimentell demonstriert, dä̈s die Erregung von chaotischen Transienten eng verwandt mit konventioneller Erregbarkeit ist. Dabei werden die Experimente durch numerische Simulationen ergänzt.

Es wird der Einflußexternen Gaussschen Rauschens nahe von sub- und superkritischen Hopf-Bifurkationen untersucht. Rausch-induzierte Schwingungen tauchen als verrauschte Vorläufer in Form von lorentzförmigen Spitzen im Powerspektrum auf. Der Kohärenzfaktor, definiert durch das Produkt aus Höhe der Spitze und Qualitätsfaktor, zeigt für beide Typen von HopfBifurkationen ein nichtmonotones Verhalten mit einem ausgeprägten Maximum bei einer bestimmten Rauschintensität. Damit wird Kohärenzresonanz experimentell demonstriert. Die Messungen zeigen neben diesen übereinstimmungen auch qualitative Unterschiede zwischen den beiden Fällen. Während die Breite des rauschinduzierten Vorläufers im Falle der superkritischen Bifurkation monoton wächst, durchläuft sie im subkritischen Fall ein ausgeprägtes Minimum. Die experimentellen Ergebnisse werden mittels eines allgemeinen 
Modells für rauschgetriebene Bewegungen in der Nähe von Bifurkationen untersucht.

Schlagwörter:

Nichtlineare dynamik, Koherenz resonance, Erregbarkeit, Chaos 


\section{Dedication}

To Ekaterina, Tatyana, Valeriya. 


\section{Contents}

1 Introduction $\quad 2$

2 The regime of ultrashort optical feedback 4

2.1 Introduction . . . . . . . . . . . . . . . . . . . . 4

2.2 Phase tuning characteristics . . . . . . . . . . . . 6

2.3 Regions of self-pulsations . . . . . . . . . . . . . . . . . 8

2.4 Phase tuning . . . . . . . . . . . . . . . . . . . . . . . 11

2.5 Hopf bifurcations . . . . . . . . . . . . . . . . . . . . 13

2.6 Theoretical Analysis . . . . . . . . . . . . . . . . . . . . . 16

2.7 Period doubling . . . . . . . . . . . . . . . . . . . . . . . . 19

3 Chaotic motion $\quad 22$

3.1 Quasi-periodic route to chaos . . . . . . . . . . . . 22

3.2 Numerical experiment . . . . . . . . . . . . . . . . . 25

3.3 Conclusion . . . . . . . . . . . . . . . . . . . . . . . . . 29

4 Excitability of Chaotic Transients 30

4.1 Chaos in crisis . . . . . . . . . . . . . . . . 30

4.2 Excitability . . . . . . . . . . . . . . . 31

4.3 Excited chaotic transients . . . . . . . . . . . . . . . . . . . . . . . . . . 36

4.4 Influence of noise . . . . . . . . . . . . . . . . . 39

4.5 Conclusion . . . . . . . . . . . . . . . . . . . . 39

5 Coherence resonance at a Hopf bifurcation 41

5.1 Noise induced regularity . . . . . . . . . . . . . . . . . . 41

5.2 Experimental characterization of bifurcations and states . . . 43

5.2.1 Experimental setup . . . . . . . . . . . . . . 43

5.2 .2 Noisy precursors . . . . . . . . . . . . . . 44

5.3 Coherence resonance close to a Hopf bifurcation . . . . . . . . 44

5.4 Generic model . . . . . . . . . . . . . . . . . . . . 47

5.5 Conclusion . . . . . . . . . . . . . . . . . 51 
6 Conclusions $\quad 52$

$\begin{array}{ll}\text { A Probability distribution function } & 54\end{array}$

B Travelling-Wave-Model $\quad 56$

$\begin{array}{ll}\text { C Device parameter in simulation } & 58\end{array}$ 


\section{List of Figures}

2.1 Scheme of a feedback setup. $\kappa$ - feedback strength, $\phi$ - feedback phase, $\tau$ - feedback delay time . . . . . . . . . . . .

2.2 Schematics of the multi-section laser device and the experimental setup. PD - photo diode, ESA - power spectrum analyzer, EDFA - erbium doped fiber amplifier, OSA - optical spectrum analyzer, Osc - digitizing sampling oscilloscope, SL - Alcatel laser, EPG - electrical pulse generator. . . . . . 7

2.3 Phase tuning properties of the passive section. Phase shift versus phase current deduced from the ASE spectra. . . . . 8

2.4 Characteristics of the most prominent types of laser emission. Left column: power spectra, middle column: optical spectra, right column: histograms. Upper row: $\mathrm{CW}$ emission (point CW of Fig. 2.5, $I_{D F B}=65 \mathrm{~mA}, I_{P}=45 \mathrm{~mA}$ ). Middle row: undamped $\mathrm{RO}$ pulsation (point RO of Fig. 2.5, $I_{D F B}=50$ $\mathrm{mA}, I_{P}=28 \mathrm{~mA}$ ). Lower row: MB pulsation (point $\mathrm{MB}$ of Fig. 2.5, $\left.I_{D F B}=70 \mathrm{~mA}, I_{P}=11 \mathrm{~mA}\right)$. . . . . . . . 10

2.5 Self-pulsation regions in the plane of phase and DFB current. The gray levels code the frequency of the major peak in the RF spectrum. White: CW emission (major RF peak less than $5 \mathrm{~dB}$ above noise floor). Diamonds: points of operation presented in Fig. 2.4.

2.6 Control of the dynamics by feedback phase. Panel a) mean output power $P$. Panel b) shift $\Delta \lambda$ of the main peak in the optical spectrum Panel c) modulation depth $M$ (difference between maximum and minimum on the sampling oscilloscope). Solid: increasing $I_{P}$. Dotted: decreasing $I_{P}$. The phase $\phi$ (bottom scale) is determined from the phase current (top scale). The DFB current is kept at $50 \mathrm{~mA}$. . . . . . . 12 
2.7 a) Characteristics of the Hopf-bifurcation of RO self-pulsations. DFB current at $50 \mathrm{~mA}$. Small circles: modulation depth. Full circles: half-width $\pi \Delta \nu$ of the resonance line in the rf spectrum. Open circles: damping rate $\tau_{d}^{-1}$ of relaxation oscillations. Both linewidth and decay rate approach zero. The horizontal offset of the decay rates is possibly due to a change of the point of operation caused by the external excitation. b) rf spectrum, c) time decay of relaxation oscillations, d) set of histograms with a change of the phase current. . . . . . . . . . 14

2.8 a) Characteristics of the Hopf-bifurcation of mode-beating selfpulsations. DFB current at $70 \mathrm{~mA}$ Small circles: modulation depth. Full circles: half-width $\pi \Delta \nu$ of the resonance line in the rf spectrum. Open circles: damping rate $\tau_{d}^{-1}$ of relaxation oscillations. b) Evolution of histograms with phase current, solid line to guide eyes. . . . . . . . . . . . . . 15

2.9 Modes calculated from Eqs. (2.8) to (2.10). Curves of constant feedback ( $K=0.1$ dashed, $K=0.3$ solid) and curves of constant phase (thin grey, 10 lines per phase period) are drawn in all panels. Panels a) and b): External cavity modes in the damping-wavelength plane, carriers fixed to the threshold density of the solitary DFB laser. Panel c): Compound cavity modes in the wavelength-density plane $\operatorname{Im}(\Omega)=0$. The carrier-induced shift $\lambda_{0}(N)$ is subtracted here for clarity. Gray area: regions of instability. . . . . . . . . . . . 18

2.10 Power spectra (left panel) and histograms for corresponding states (right panel). Current in DFB section is constant $I_{D F B}=90$ $\mathrm{mA}$. From (a) to (d) phase current $I_{P H}$ takes the values 18.6 $\mathrm{mA}, 19 \mathrm{~mA}, 19.2 \mathrm{~mA}, 20 \mathrm{~mA}$. An arrow indicates the peak at the frequency $3 f_{0} / 4 \ldots \ldots \ldots \ldots$

3.1 Schematic of an AFL. An additional amplifier section enables the control of feedback strength. The different sections are controlled by independent currents. ESA - power spectrum analyzer, EDFA - erbium doped fiber amplifier, Osc - digitizing sampling oscilloscope. . . . . . . . . . . . .

3.2 Upper panel: maxima and minima of laser intensity vs. current in the phase section $I_{P H}$. Evolution of states, first and third columns: power spectra, second and fourth column: probability distribution function - histogram. From (a) to (j) $I_{P H}$ takes values $1.5 \mathrm{~mA}, 6 \mathrm{~mA}, 6.8 \mathrm{~mA}, 7 \mathrm{~mA}, 7.3 \mathrm{~mA}, 7.5 \mathrm{~mA}$, $7.7 \mathrm{~mA}, 7.9 \mathrm{~mA}, 8.05 \mathrm{~mA}, 8.2 \mathrm{~mA} \ldots \ldots . . . . . .24$ 
3.3 Maxima and minima of output power versus phase $\phi . \quad \mathrm{H}$ Hopf bifurcation, T - torus bifurcation. . . . . . . . . 26

3.4 Changes of the dynamics when the phase $\phi$ varies, $I_{D F B}$ and $I_{A}$ are constant and equal $41.7 \mathrm{~mA}$ and $49.5 \mathrm{~mA}$, respectively. Shown are the phase space projections in the plane of power from left facet vs $n_{D F B}$ (first column), the time trace (second column), the power spectrum (third column). From (a) to (e) $\phi / 2 \pi$ takes the values $0.88,0.8,0.7,0.688,0.66$. Note the different scales. . . . . . . . . . . . . . . 27

4.1 a) Schematic phase-space portrait of an excitable chaotic saddle. $u_{1}, u_{2}$ - the two branches of unstable manifold of the saddle-focus. b) Laser with external excitation. ESA - electrical spectrum analyzer, EPG - electrical pulse generator, PD - photo diode, EDFA - Erbium doped fiber amplifier, SL - gain-switched DFB laser. . . . . . . . . . . . . . . . . 32

4.2 Left panel: power spectra, right panel histograms at selected phase currents, chaos $I_{P H}=6.85 \mathrm{~mA}$ (solid gray lines), stationary $\mathrm{cw}$ state $I_{P H}=6.9 \mathrm{~mA}$ power spectrum (dotted), histogram (grey dotted). Power spectrum (black solid line) and histogram (dark grey area) at $I_{P H}=6.9 \mathrm{~mA}$ under the superthreshold stimulation. Note the logarithmic scale in both plots. . . . . . . . . . . . . . . . 34

4.3 Response of the multi-section laser on 30 ps optical excitation. Maximum device output versus optical pulse energy $\left(I_{P}=6.9\right.$ $\mathrm{mA}) \ldots \ldots \ldots \ldots \ldots \ldots \ldots$ 
4.4 Excitability threshold versus distance from the boundary crisis.

a) Calculation $\left(I_{D F B}=40 \mathrm{~mA}, I_{A}=28 \mathrm{~mA}\right)$. SN bifurcation $(\odot)$ and boundary crisis occur at feedback phase $\phi=4.1936$ and $\phi_{c}=4.1930$, respectively $\left[\mathrm{UKR}^{+} 06\right]$. Rectangles: stimulus energy at threshold, scattering dots left of $\phi_{c}$ : maximum device output in the chaotic regime computed over a time of $10 \mathrm{~ns}$, lower solid and dashed lines: carrier inversion $\Delta n$ on node and saddle, respectively, measured relative to its value at the SN bifurcation. b) Measurement $\left(I_{D F B}=41.7 \mathrm{~mA}\right.$, $I_{A}=49.5 \mathrm{~mA}$ ). Meaning scattering dots as in a), circles and rectangles: maximum spike strength and stimulus energy at threshold under superthreshold stimulation are inserted from Ref. [Bet03].

Note that the power output right after the device edge is plotted in the calculation, while the experimental signal is attenuated by the various optical elements of the setup before detection. . . . . . . . . . . . . . .

4.5 Transients after superthreshold excitation. a) Comparison of two calculated responses (black and grey, $\phi=4.15$ ). The stimuli differ only by the initial phase difference between internal and injected fields. Initially, the two transients are indistinguishable. At later times, however, they diverge resembling the extreme sensitivity of chaos on initial conditions. One transient (grey) approaches equilibrium after $3 \mathrm{~ns}$ ). The other one stays nearly 20 ns on the chaotic ruin. b) Superposition of 500 measurements with the sampling oscilloscope (stimulus repetition rate $12.5 \mathrm{MHz}$ ). The point of operation (phase current $6.9 \mathrm{~mA}$ ) is close to the crisis. and not all transients do return to the rest state within the $80 \mathrm{~ns}$ repetition period. c) Probability of presence in the CS versus time after stimulation for three different distances from the boundary crisis $I_{P}-I_{c}$ : $0.04 \mathrm{~mA}$ (triangles), $0.02 \mathrm{~mA}$ (squares), and $0.01 \mathrm{~mA}$ (circles). Lines: singe-exponential fits.

4.6 Mean lifetime $\tau$ on the CS versus $I_{P}-I_{c}$. Solid line: inversepower function with exponent $\gamma=1 . \ldots . . . . . .38$

4.7 Histogram of stationary state close to the boundary crisis $I-$ $I_{C} \approx 0.01 \mathrm{~mA}$, Gaussian approximation (solid line). Side wings indicate noise induced wandering on the chaotic saddles. Ratio of area under the wings and the main peak is $0.6 \times 10^{-4}$. 
5.1 a) schematics of the multi-section laser device and the experimental setup. NS - an electrical noise source, Att. - a tunable attenuator, T - a bias tee, PD - a photo diode, ESA - a power spectrum analyzer, EDFA - an erbium doped fiber amplifier. b) a power spectrum of electrical broadband noise; c) an intensity distribution of noise (open dots), Gaussian approximation (solid line). . . . . . . . . . . . . . . . . . 43

5.2 Left column: maximum $\left(P_{\max }\right)$ and minimum $\left(P_{\min }\right)$ output power versus phase current. Right column: power spectra of the device under the influence of noise. (a2) Before the supercritical Hopf bifurcation without external noise (lower plot) and at optimum noise level (upper plot), solid lines are fits with a Lorentzian shape $\left(I_{D F B}=70 \mathrm{~mA}, I_{A}=5.62 \mathrm{~mA}\right.$, $\left.I_{P H}=11.8 \mathrm{~mA}\right)$. The same for the subcritical case (b2) $\left(I_{D F B}=70\right.$ $\left.\mathrm{mA}, I_{A}=30.6 \mathrm{~mA}, I_{P H}=98.2 \mathrm{~mA}\right)$ and the torus bifurcations (c2) $\left(I_{D F B}=41.7 \mathrm{~mA}, I_{A}=47 \mathrm{~mA}, I_{P H}=6.1 \mathrm{~mA}\right)$. Arrows correspond to operating points.

5.3 Peak height (gray dots), normalized width (open dots), and signal-to-noise ratio (black dots) versus noise intensity as deduced from the power spectra by Lorentzian fits. Note the log scale. (a1,2) Supercritical bifurcation, (b1,2) Torus bifurcation, and $(\mathrm{c} 1,2)$ Subcritical bifurcation. Maxima of curves are normalized to unity. . . . . . . . . . . . . 46

5.4 (a), (b) calculated trajectories. (a1), (b1) local damping F(r). Normalized distribution $P(r)$ for different level of noise. (a2) I - $\mathrm{D}=0.0001, \mathrm{II}-\mathrm{D}=0.0016$, III - $\mathrm{D}=0.008, \mathrm{IV}-\mathrm{D}=0.13$. (b2) I - $\mathrm{D}=0.0001$, II $-\mathrm{D}=0.0016$, III $-\mathrm{D}=0.008$, IV $-\mathrm{D}=0.0625$; Left column: supercritical case; Right column: subcritical case. . . 48

5.5 Results of numerical simulation: spectral width $\Delta \omega$ (dots) and mean local damping $\langle F(r)\rangle$ (squares); a) supercritical Hopf bifurcation, b) subcritical Hopf bifurcation. . . . . . . . . 50

B.1 Schematic of a DFB laser. $E^{ \pm}(t, z)$ - the forward and backward travelling waves, $r_{0}, r_{L}$ - facet reflectivities. . . . 56 


\section{List of Tables}

C.1 Parameter values used for the DFB, phase tuning $(\mathrm{P})$, and amplifier (A) sections. Deviations are noted in the text. . . . 59 


\section{Chapter 1}

\section{Introduction}

Self-organization refers to a process in which the internal organization of a system increases spontaneously without being imprinted by an outside source. There exists a vast number of examples of self-organization in physics, chemistry, and biology. The laser is a paradigm of self-organization in open systems [Hak83a]. When the atoms of the laser material are excited or "pumped" from outside, they emit light waves. If the laser atoms are pumped only weakly by external sources, the laser acts as an ordinary lamp. The atoms independently of each other emit wavetracks with random phases. If the pumping is further increased, suddenly within a very sharp transition region the linewidth of the laser light may become of the order of one cycle per second so that the phase of the field remains correlated on the macroscopic scale.

Feedback may change dramatically the dynamics of systems. A number of new ordering effects can occur [Goo89]. Prominent examples of such systems are laser with delayed optical feedback when an external mirror is placed at a distance from the laser and reflects a part of the output back into the device. Most of the research regarding delayed optical feedback to date aims at feedback lengths from many meters down to a few centimeters. Feedback setups with these external cavity lengths are interesting because of the very complex and irregular dynamics they can provide. A practical application is the generation of chaotic optical signals for encrypted communication [Oht02]. Very recently, the regime of short feedback delay has attracted much attention due to the nontrivial dynamics [HFEG01]. A characteristic phenomenon in the short cavity regime is the occurrence of regular and irregular pulse packages $\left[\mathrm{HFE}^{+} 03\right]$.

This thesis investigates the novel regime of ultrashort feedback delay. It is achieved when semiconductor laser and feedback part are integrated on a single chip, both with the same length dimensions of a few hundred micrometers 
only. An outstanding feature of these devices is that the feedback parameters can be tuned by the injection currents. It has been shown that the phase space can be tailored by change of these injection currents [BBK $\left.{ }^{+} 04 ; \mathrm{Bau} 05\right]$. My work is dedicated to the investigation of self-organization processes in such devices, also exploiting external regular and stochastic perturbations. In Chapter 2, I experimentally verify that undamped relaxation oscillations (ROs) and mode beating (MB) oscillations are the generic pulsation types for the laser with ultra-short optical feedback. Also is shown that both types of pulsation appear in a Hopf bifurcation $\left[\mathrm{UBB}^{+} 04\right.$; UBOBH04]. Chapter 3 is devoted to the appearance of chaotic dynamics. The quasi-periodic route to chaos, which results in a high-dimensional torus-like chaotic attractor is detected. Furthermore, a sudden destruction of chaotic motion is experimentally and numerically observed. A global bifurcation, a boundary crisis $(\mathrm{BC})$, is responsible for the destruction of the chaotic attractor.

Chapter 4 combines two nonlinear dynamical phenomena, excitability and chaotic transients, treated so far only separately. Excitability means that the response of a system on external perturbations is "all" or "none" depending on whether the strength of the stimulus is above or below a critical threshold. Prominent examples of excitable systems are the spiking of neurons [Hod47], the cardiac muscle [Mur93], the dynamics of life populations [CB99], or nonlinear chemical reactions [ZZ70]. On the other hand chaotic transients (CT), usually appearing behind $\mathrm{BC}$, are long episodes of chaotic behavior which end eventually at an attractor that is usually not chaotic. In my experiments, high-dimensional CT are excited by short optical pulses. The excitation of the CT exhibits a distinct threshold as well as a refractory time and, sufficiently far from the boundary crisis, the standard response of excitable systems is observed [ $\left.\mathrm{UKR}^{+} 06\right]$.

In Chapter 5, I study the impact of external stochastic perturbation on the dynamics of the laser, close to a Hopf bifurcation. Noise induces oscillations near an onset of a bifurcation [EHRSG86; Wie85]. The regularity of these oscillations can be improved, and coherence resonance [PK97; GDNH93; NSS97] is detected. The noise improves the signal-to-noise ratio [GDNH93; NSS97]. However, while the existence of an optimum noise level is a common feature for both types of Hopf bifurcations, the physics behind the resonance effect is qualitatively different. In the supercritical Hopf bifurcation, the damping is a monotonic function of the amplitude and, hence, of the noise. The subcritical case is distinguished by a nonmonotonic relation between the local damping and the noise intensity. These results are published in $\left[\mathrm{UWFH}^{+} 05 ; \mathrm{KLGZ06}\right]$. Finally, Chapter 6 summarizes my thesis with conclusions. 


\section{Chapter 2}

\section{The regime of ultrashort optical feedback}

\section{$2.1 \quad$ Introduction}

The single-mode laser is a paradigm of self-organization in dissipative systems [Hak83a]. At threshold it undergoes a transition from incoherent to coherent emission. One optical mode becomes undamped here in a Hopf bifurcation. Its contribution to the optical field is harmonically oscillating and dominates beyond threshold, giving rise to high coherence. Distributed feedback (DFB) semiconductor lasers come close to this ideal [CWP98]. They exhibit continuous wave $(\mathrm{CW})$ single mode emission up to high pump currents. Upon a perturbation, the intensity returns back to the stationary $\mathrm{CW}$ value with well damped relaxation oscillations (RO). Optical feedback can destabilize the CW state [LK80]. Phenomena like self-sustaining intensity pulsations, coherence collapse [FHME98], and others have been recently predicted and experimentally observed. Potential applications cover high-speed data transmission, cryptography [Oht02], etc.

Optical feedback is usually achieved by combining the laser with an external mirror, see Fig.2.1. Three parameters are characterizing the feed-

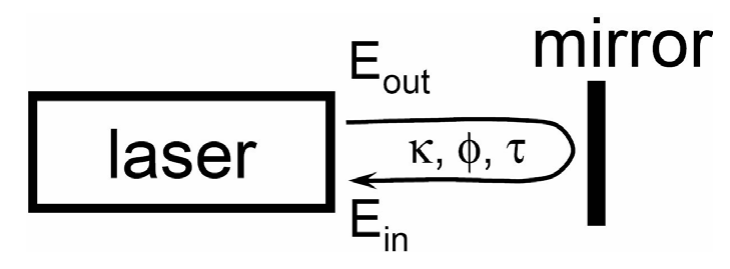

Figure 2.1: Scheme of a feedback setup. $\kappa$ - feedback strength, $\phi$ - feedback phase, $\tau$ - feedback delay time 
back. First, the feedback strength $K$ gives the ratio of in- and outgoing field amplitudes $\left|E_{\text {in }} / E_{\text {out }}\right|$ at the laser facet. The second parameter is the feedback delay time $\tau=2 L / c$, which depends on the feedback cavity length $L$. Together with the lasing frequency $\omega_{0}$ of the solitary laser it gives the third parameter, the feedback phase $\phi=\omega_{0} \tau$.

Theoretically, two approaches have been used, in order to investigate the delayed feedback configuration [WT02]. One of them is the full travellingwave equations (TWEs) $\left[\mathrm{RWS}^{+} 00\right]$. The resulting TWEs can be solved numerically, e.g. with the LDSL simulation tool [KRWH03]. This allows to regard effects like gain-compression, gain-dispersion and arbitrary feedback strength. Although giving a detailed description of the experimental situation [KRWH03; $\mathrm{BBK}^{+}$04], this approach has one important disadvantage: basic mechanisms are hidden by the complexity of the model.

For the understanding, the simple rate equations are commonly used in order to qualitatively describe the dynamical phenomena. In a phenomenological approach, the equation for the optical field can be supplemented by an additional feedback term responsible for the re-injected light. This results in the mean-field rate equation in normalized form

$$
\begin{aligned}
\frac{d}{d t} E(t) & =\frac{1}{2}\left(1+i \alpha_{H}\right) N E(t)+\eta e^{-i \phi} E(t-\tau), \\
\frac{d}{d t} N(t) & =\epsilon\left(J-N-(N+\nu)|E(t)|^{2}\right) .
\end{aligned}
$$

for the field of slowly varying amplitude $E$ and the carrier density $N$ with $\alpha_{H}$ as the Henry factor, $\phi$ as the feedback phase and $\eta$ as the effective feedback strength. $\epsilon=\tau / \tau_{c}$ is small with respect to the other coefficients, where $\tau$ is feedback time and $\tau_{c}$ is a carrier lifetime. $J$ stands for the pumping current and $-\nu$ corresponds to the transparency density. These equations were originally proposed by Lang and Kobayashi (LK) [LK80] for the description of feedback effects in semiconductor lasers. By the feedback term the laser equations are transformed into delay differential equations, which in general have high degree of complexity due to infinite dimensional phase space [Kra00]. The LK equations are widely used to describe lasers with feedback. A large number of investigations have shown that this approach gives a good description in the long- and short-cavity regimes [MTM92; $\mathrm{HFE}^{+} 03$; WT02].

The behavior in presence of feedback crucially depends on the number of modes that are of relevance in the compound device. The modes of compound cavity are the steady-state continuous wave type solutions of Eqs.2.1 - The number of modes grows when the feedback strength $K$ increases. A second factor arises through the time-scales involved. Photon life-times in typical semiconductor lasers are $\tau_{P} \approx 1-10 \mathrm{ps}$, while the period of the relax- 
ation oscillations $\tau_{R}$ ranges between 0.1 and 1 ns. In the long-cavity limit, addressed in most previous studies, $\tau$ is much longer than $\tau_{R}$. The solitary mode is hence transformed in a quasi-continuous spectrum of external cavity modes, even for modest $K$. The consequence is an irregular dynamical response with stochastic power dropouts. Studies on shorter cavities have yielded qualitatively different behavior [LK80; HFEG01; $\mathrm{HFE}^{+}$03]. Here, the feedback phase begins to influence the field-inversion dynamics in the laser. Regular intensity pulsations have been reported already in early studies [LK80]. However, the multimode nature of the used Fabry-Perot lasers mixes with feedback effects [LK80]. Using single mode lasers in a short feedback regime $\left(\tau / \tau_{R} \approx 0.3\right.$ to 1$)$, pulse packages have been observed recently, that originate from a global trajectory along a limited number of modes in the phase-inversion space.

When I started my work, the metamorphosis of this scenario with even shorter delay time was not subject to experiments. An exception are DFB lasers with active feedback used to generate self-pulsations of several ten $\mathrm{GHz}$ $\left[\mathrm{BBK}^{+} 02\right]$. The dynamics of these devices is however modified by coupling of the photons to two independent inversion ensembles $\left[\mathrm{BBK}^{+} 04\right]$. Only the limit of feedback with zero delay is well known - the reflectivity from cleaved facets does not cause dynamic instabilities in DFB lasers.

In this chapter experimental revealing of basic self organization processes in laser with ultrashort optical feedback is reported. The main results have been published in Ref.[ $\left.\mathrm{UBB}^{+} 04\right]$. In extension of these inventions, possible transitions to chaotic operation are characterized, which have been obtained more recently.

\subsection{Phase tuning characteristics}

The investigated device is sketched in Fig. 2.2. It is based on the InGaAsPInP material system and the optical wave is guided by a ridge waveguide structure. It consists of two sections: a $L_{D F B}=220 \mu \mathrm{m}$ long DFB laser is integrated with a passive feedback section of length $L_{P}=200 \mu \mathrm{m}$. The active bulk $\lambda_{\text {gap }}=1.55 \mu \mathrm{m}$ layer of the DFB laser is embedded in an asymmetric $\lambda_{\text {gap }}=1.3 \mu \mathrm{m}$ InGaAsP optical waveguide which has an index coupled grating without phase shifts. A coupling coefficient $\kappa=130 \mathrm{~cm}^{-1}$ was chosen in order to prevent mode switching between the two stop-band sides of the DFB laser. The short wavelength mode is supported by the resulting longitudinal hole burning. The facet of the DFB section is anti-reflection (AR) coated with a remaining power reflectivity of $10^{-4}$. In the feedback section the $1.55 \mu \mathrm{m}$ layer is removed and the remaining layers do not directly couple 
to the emission of the laser. Its rear facet is cleaved, with a resulting power reflectivity $R \approx 0.3$. The feedback parameters are

$$
\begin{aligned}
\phi & =-\frac{4 \pi}{\lambda} n_{e f f} L_{P} \\
K & =T_{A P} \exp \left(-\alpha_{P} L_{P}\right) \sqrt{R} \\
\tau & =2 L_{P} v_{g}^{-1}
\end{aligned}
$$

The phase shift $\phi$ is proportional to the effective refractive index $n_{\text {eff }}$ which in turn is affected by the carriers injected into this section. Therefore, the current $I_{P}$ can be used for phase tuning at fixed laser parameters in contrast to previous studies with external feedback cavity, exploiting laser current [LK80] (and references therein) or laser temperature [HFEG01; $\mathrm{HFE}^{+} 03$ ].

The device is produced in group of Bernd Sartorius in the Heinrich-HertzInstitut für Nachrichtentechnik (HHI) ${ }^{1}$, Berlin. In detail, the technical characteristics of the laser is investigated in Ref. thesis [Bro05]. Measured amplitude spontaneous emission (ASE) spectra are used from Ref. [Bro05] in order to estimate the phase tuning characteristics of the device. The extracted data are shown in Fig.2.3. More than 3 phase periods are accessible before the phase shift saturates beyond $80 \mathrm{~mA}$. A secondary effect of $I_{P}$ is induced free-carrier absorption. It degrades the feedback strength $K$ by roughly a factor 3 in the considered range of currents. Comparing with transmission spectra of non-biased isolated feedback sections cut from the same wafer, we estimated about $10 \mathrm{~cm}^{-1}$ background losses in the feedback cavity and a transmissivity $T_{A P} \approx 0.7$ of the active-passive interface. The corresponding feedback amplitudes range from $K \approx 0.3$ down to $K \approx 0.1$. The measured group index $c / v_{g} \approx 3.5$ is nearly independent of the phase current, yielding a constant delay $\tau \approx 5 \mathrm{ps}$. The according round-trip frequency $\tau^{-1} \approx 200 \mathrm{GHz}$

\footnotetext{
${ }^{1}$ Since 2003: FhG-HHI
}

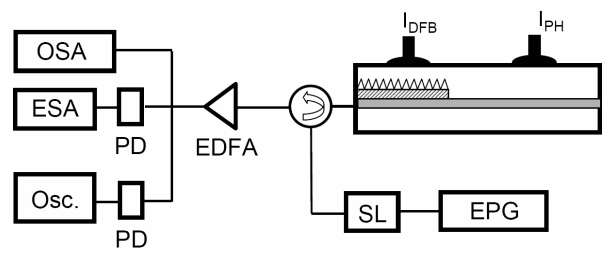

Figure 2.2: Schematics of the multi-section laser device and the experimental setup. PD - photo diode, ESA - power spectrum analyzer, EDFA - erbium doped fiber amplifier, OSA - optical spectrum analyzer, Osc - digitizing sampling oscilloscope, SL - Alcatel laser, EPG - electrical pulse generator. 
is nearly two orders of magnitude larger than typical relaxation oscillation frequencies and, hence, the present device realizes the limit of very short feedback cavities.

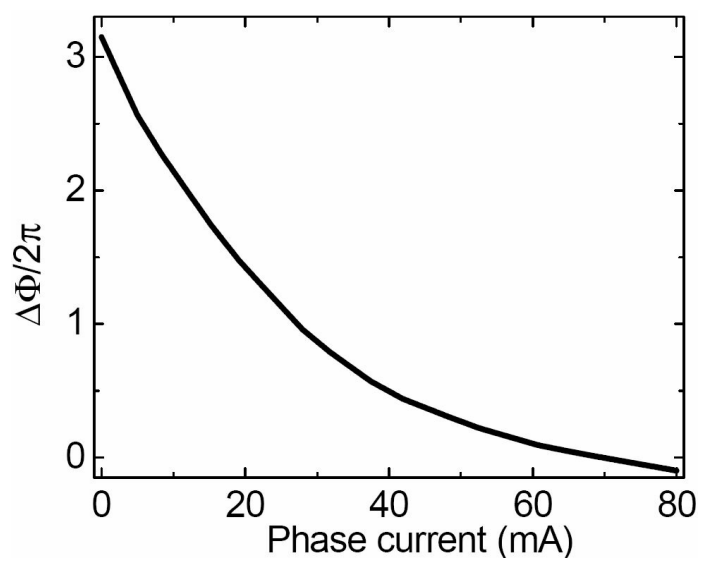

Figure 2.3: Phase tuning properties of the passive section. Phase shift versus phase current deduced from the ASE spectra.

The following experimental setup is used in order to detect and to characterize self-pulsations. The two section laser is connected to an oscilloscope, an optical spectrometer and an electrical spectrum analyzer by a single mode optical fiber (SM Patchcord 9/125) from the DFB facet. The signal is amplified by the Erbium Doped Fiber Amplifier (EDFA). To obtain time series the sampling oscilloscope(HP 54120B Digitize Oscilloscope, bandwidth of $50 \mathrm{GHz}$ ) with $\mathrm{u} 2 \mathrm{t}$ photo diode is used. The optical spectra are measured by a Czerny-Turner spectrometer (THR 1500, grating $600 \mathrm{~g} / \mathrm{mm}$ ) with an IR camera as detector. Power spectra are acquired by an electrical spectrum analyzer (R\&S FSP $9 / 40 \mathrm{GHz}$, bandwidth of $40 \mathrm{GHz}$ ) with u2t photo diode. The time averaged mean power is measured by an optical power-meter (NOYES OPM 5), with averaging time of $5 \mathrm{~s}$. Measurements are carried out at varying currents in the passive and the active sections. Currents are controlled by the current source (Tektronix/Profile PRO 8000). Both sections of the laser are kept at a constant temperature $20 C^{o} \pm 0.05 C^{o}$.

\section{$2.3 \quad$ Regions of self-pulsations}

The dynamics of the laser with ultrashort optical feedback strongly depends on the feedback intensity. For low feedback strengths, the experimental situation is similar to a solitary laser. Once the feedback is strong enough that a pair of longitudinal modes can coexist, additional dynamics is induced by 
beating phenomena. Mode beating (MB) requires two longitudinal modes which are simultaneously lasing. This corresponds to the configuration first investigated by Tager and Petermann [TP94]. The most important finding of their work was a stable coexistence of mode and antimode, provided sufficiently strong feedback and an appropriate feedback phase. These results were later confirmed by analytical investigations in Refs. [ERGK00; WT02].

Below the feedback strength required for MB only single-mode dynamics occurs. An analysis of the short cavity regime in [TP94] has shown that the single-mode scenario can give rise to self-sustained pulsations. These oscillations are similar as in multisection lasers comprising two detuned gratings $\left[\mathrm{TWS}^{+} 00\right]$. The setup in these devices involves a DFB mode positioned spectrally on a decreasing reflectivity slope of the other DFB section. Mediated by $\alpha_{H}$, any fluctuation in the lasing DFB section leads to a variation of the wavelength. In presence of dispersive feedback this induces a change of the feedback strength, i.e. the resonator quality of the cavity is modified. For this reason, the effect is also called dispersive Q-switching (DQS). As a result of DQS, periodic modulations of the laser intensity or undamped ROs are observed; frequencies are slightly enhanced compared to the damped ROs of a solitary laser.

In order to find the theoretically predicted MB and URO self-pulsations [TP94; WT02; ERGK00; $\mathrm{TWS}^{+} 00$ ] we map the two injection currents over a wide range. Depending on the point of operation, different types of RF and optical spectra are observed. Fig. 2.4 shows characteristic examples. A single line in the optical spectrum and a flat noise floor in the RF spectrum are attributes of CW emission. Sharp and strong RF peaks and a corresponding splitting of the optical emission line into several sublines are the fingerprints of self-pulsations.

Fig. 2.5 shows those regions in the two-parameter plane $I_{D F B}-I_{P}$ where the major RF peak exceeds the noise floor by more than $5 \mathrm{~dB}$. Cycles of alternating SP and CW regimes appear in dependence on $I_{P}$ due to the phase tuning property of this current. The injection level of the DFB section has only a small influence on the position of the SP islands. This influence is due to the change of the feedback phase with the emission wavelength. The SP islands of the different cycles are similar but not identical because $I_{P}$ influences not only the phase but also the strength of feedback.

The frequencies of SP range from about $1 \mathrm{GHz}$ up to $24 \mathrm{GHz}$. They increase sub-linearly with $I_{D F B}$ in the lower part of each SP island. This behavior is a fingerprint of relaxation oscillations. We can conclude that those parts of the SP-islands, which continuously evolve from these low frequency parts are regions of undamped RO. Below we shall give more evidence for this conclusion. We shall also proof that the high-frequency parts of the islands 


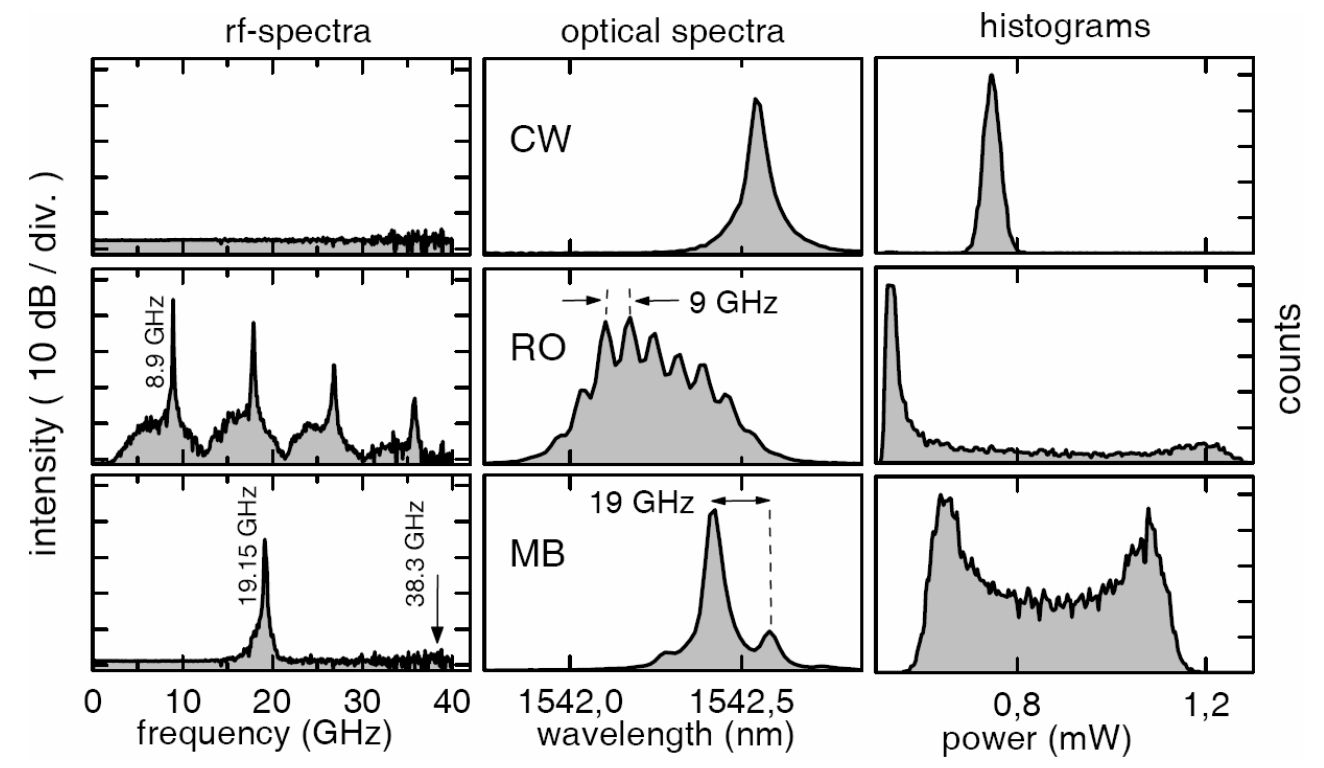

Figure 2.4: Characteristics of the most prominent types of laser emission. Left column: power spectra, middle column: optical spectra, right column: histograms. Upper row: CW emission (point CW of Fig. 2.5, $I_{D F B}=65 \mathrm{~mA}$, $I_{P}=45 \mathrm{~mA}$ ). Middle row: undamped RO pulsation (point RO of Fig. 2.5, $I_{D F B}=50 \mathrm{~mA}, I_{P}=28 \mathrm{~mA}$ ). Lower row: $\mathrm{MB}$ pulsation (point $\mathrm{MB}$ of Fig. 2.5, $I_{D F B}=70 \mathrm{~mA}, I_{P}=11 \mathrm{~mA}$ ).

are MB pulsations, in particular the upper parts within the first two phase cycles.

The right column in Fig. 2.4 shows how frequent a given optical power is detected by the oscilloscope within a persisted time. These histograms provide further statistical characteristics of the measured signals which is proportional to a probability density function (PDF), see Appendix A. The stationary state is represented by a single peak at the mean power. Its width is a measure for the noise induced intensity fluctuations. Regular pulsations are characterized by wide two-peak distributions. The peaks represent the mean minimum and maximum powers. Their heights measure how fast the intensity passes through the respective extrema. The RO histogram exhibits a high extinction ratio and a very distinct peak at the minimum power. Thus, the RO pulsations are comparatively short pulses separated by relatively long valleys with minimum intensity. This is consistent with the presence of many harmonics in the power spectra. In contrast, the extinction of MB pulsations is smaller and the two similar peaks indicate a nearly sinusoidal behavior and in power the power spectra single spike at the fundamental frequency. 


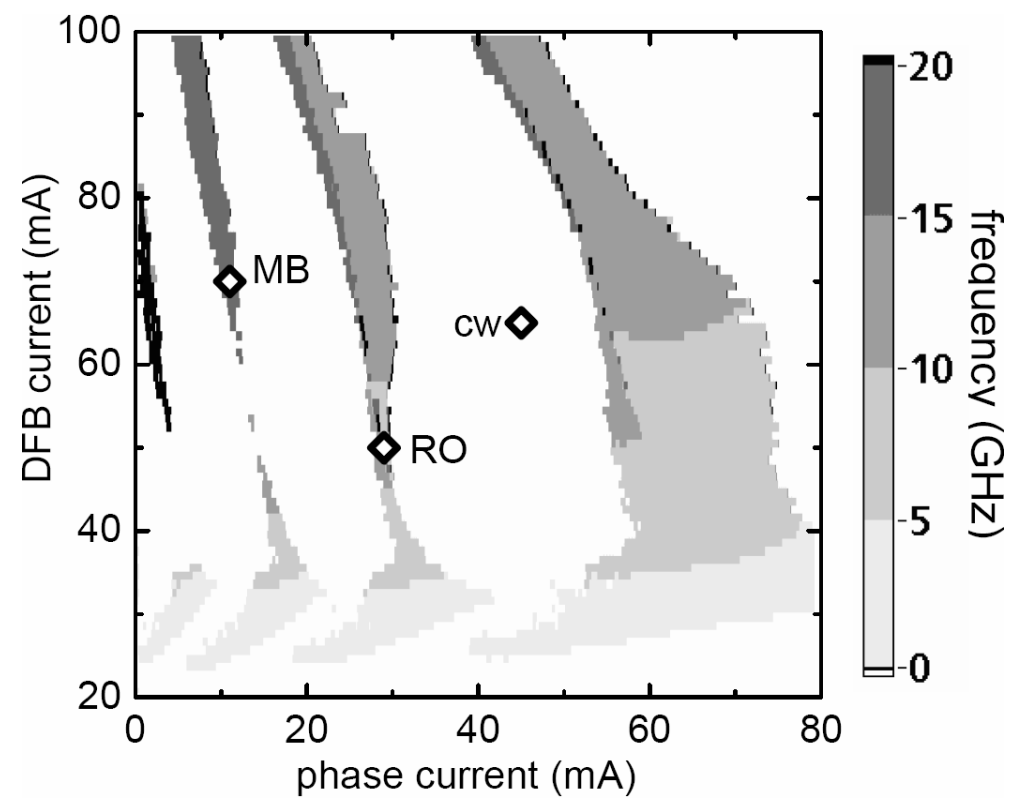

Figure 2.5: Self-pulsation regions in the plane of phase and DFB current. The gray levels code the frequency of the major peak in the RF spectrum. White: CW emission (major RF peak less than $5 \mathrm{~dB}$ above noise floor). Diamonds: points of operation presented in Fig. 2.4.

\subsection{Phase tuning}

So far we have identified the regions and frequencies of self-pulsations. The phase current appeared as main parameter for the bifurcations from $\mathrm{CW}$ to SP. Now we give a deeper characterization of the SP and of the bifurcations associated with the feedback phase. To these purposes we change the phase current in steps of $0.1 \mathrm{~mA}$ from $0 \mathrm{~mA}$ to $80 \mathrm{~mA}$ and reverse, keeping the DFB current at $50 \mathrm{~mA}$. Mean intensity, modulation depth, and wavelength of the emission from the DFB facet are recorded in each point of operation. In Fig. 2.6, the data are plotted versus the phase parameter $\phi$ determined from $I_{P}$ as described in Section 2.2.

Power and wavelength undergo cyclic variations with $\phi$ and exhibit distinct hysteresis effects due to the movement of external cavity modes across the fixed DFB resonance. The power becomes maximum when a mode coincides with the resonance. With changing $\phi$ from here, the threshold increases and the power decreases until a jump to the next better mode appears. The regions of nonzero modulation depth in Fig. 2.6c) are indications of self-pulsations. They appear for increasing phase (forward direction) in each period just before jumping to the next mode. Similar power and wave- 


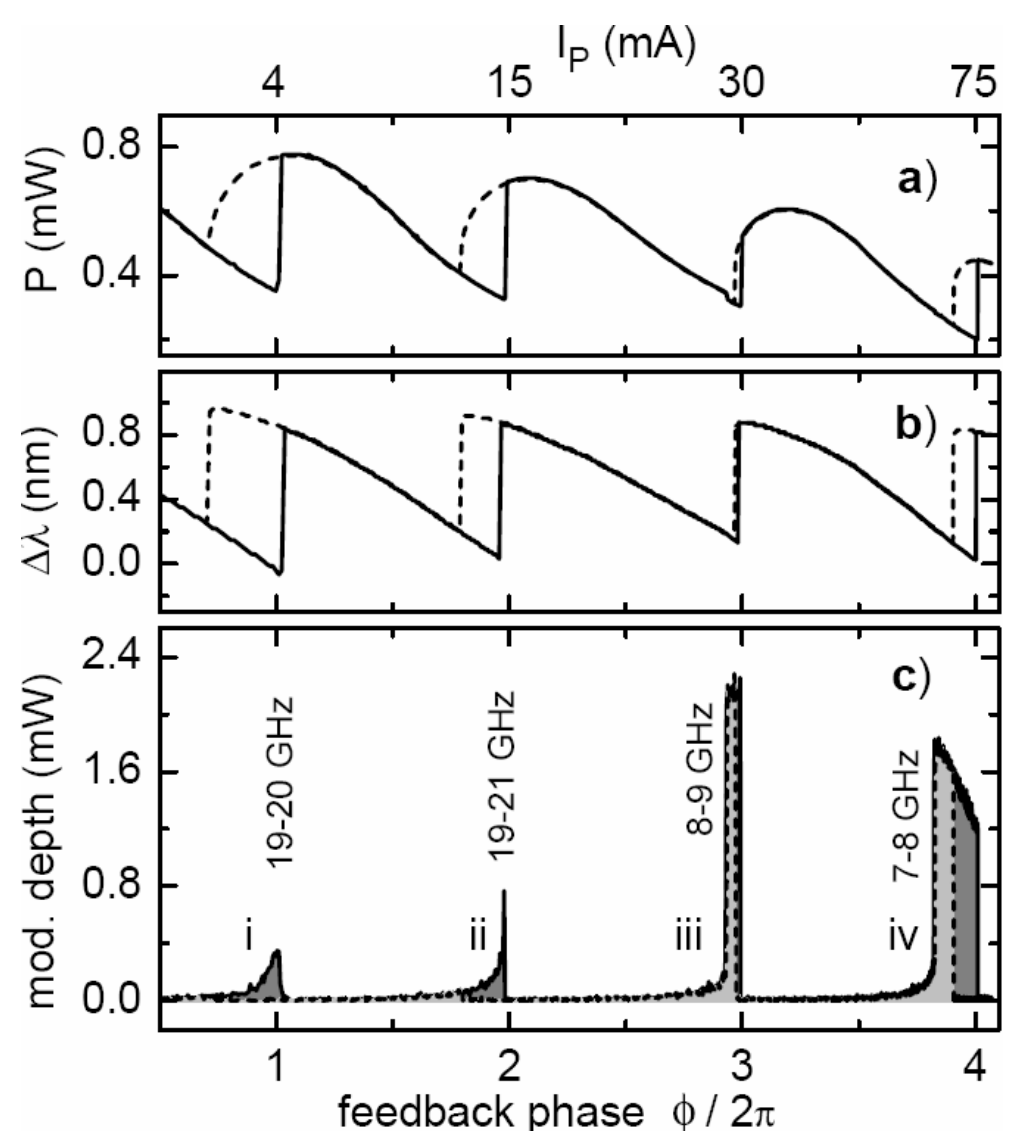

Figure 2.6: Control of the dynamics by feedback phase. Panel a) mean output power $P$. Panel b) shift $\Delta \lambda$ of the main peak in the optical spectrum Panel c) modulation depth $M$ (difference between maximum and minimum on the sampling oscilloscope). Solid: increasing $I_{P}$. Dotted: decreasing $I_{P}$. The phase $\phi$ (bottom scale) is determined from the phase current (top scale). The DFB current is kept at $50 \mathrm{~mA}$.

length undulations were observed when changing the injection current of a semiconductor laser subject to external optical feedback [LK80].

The different periods are not completely equivalent because $I_{P}$ changes not only the phase of the feedback but also its strength $K$ from $\approx 0.3$ down to $\approx 0.1$ in the investigated current range (see Section 2.2). Obviously, hysteresis and pulsation effects are most sensitive to this effect. They qualitatively change from region ii to region iii.

In regions $\mathrm{i}$ and ii pulsations occur only with small amplitude in a small interval in forward direction and the frequencies are around $20 \mathrm{GHz}$ as expected for MB pulsations. In the other two periods, the SP have distinctly 
smaller frequencies, higher amplitudes, and show less hysteresis.

\subsection{Hopf bifurcations}

Theory predicts that SPs appear in Hopf bifurcation [WT02]. Mathematically a Hopf bifurcation is characterized by a pair of complex conjugated eigenvalues of the dynamical matrix moving across the imaginary axis. What does it mean from physical point of view? Among many vibrational modes there is one oscillator whose damping approaches zero. In order to detect this process experimentally, we can profit from the noise in our real system, which is omnipresent e.g. due to spontaneous emission. Noise drives all possible oscillations. If the damping of one oscillator approaches zero, its oscillation amplitude increases. An increasing and narrowing line should appear in the power spectrum as a precursor of the Hopf bifurcation, whose position and width are a direct measure for the relevant complex eigenvalues.

First we apply this concept to the SP region iii of Fig. 2.6. The modulation depth re-plotted in a zoomed scale exceeds the noise level when approaching the pulsation region from left (see Fig. 2.7). This amplitude increase is already the fingerprint of an oscillator getting undamped. At the same time, a well resolvable line in the rf spectra appears. The distinct decay of its width gives evidence of a Hopf bifurcation at this boundary of the self-pulsation region.

In order to evaluate the physical nature of the undamped oscillator, the laser was excited by an external pulse and the decaying response was measured (see inset of Fig. 2.7). The decay rates fall with the same slope as the rf linewidths. Thus, the oscillations undergoing the Hopf-bifurcation are relaxation oscillations. The small deviation between measured resonance widths and decay rates is possibly due to the small impact of the external excitation on the feedback phase.

The frequency of the decaying oscillations is $12 \mathrm{GHz}$ in agreement with the rf-peak positions, distinctly above the $\mathrm{RO}$ resonance of comparable DFB lasers without feedback. This finding is a typical effect of undamping by dispersive Q-switching which is accompanied by an increase of the effective differential gain [WBWR96; $\mathrm{TWS}^{+} 00$ ].

The modulation depth suddenly blows up when crossing the bifurcation accompanied by a slowing down of the oscillations from $12 \mathrm{GHz}$ to $9 \mathrm{GHz}$. However, no hysteresis is observable within the experimental resolution. We conclude, the investigated point of operation is very close to a codimension two degenerate Hopf bifurcation, where the Hopf changes its character from subcritical to supercritical. 


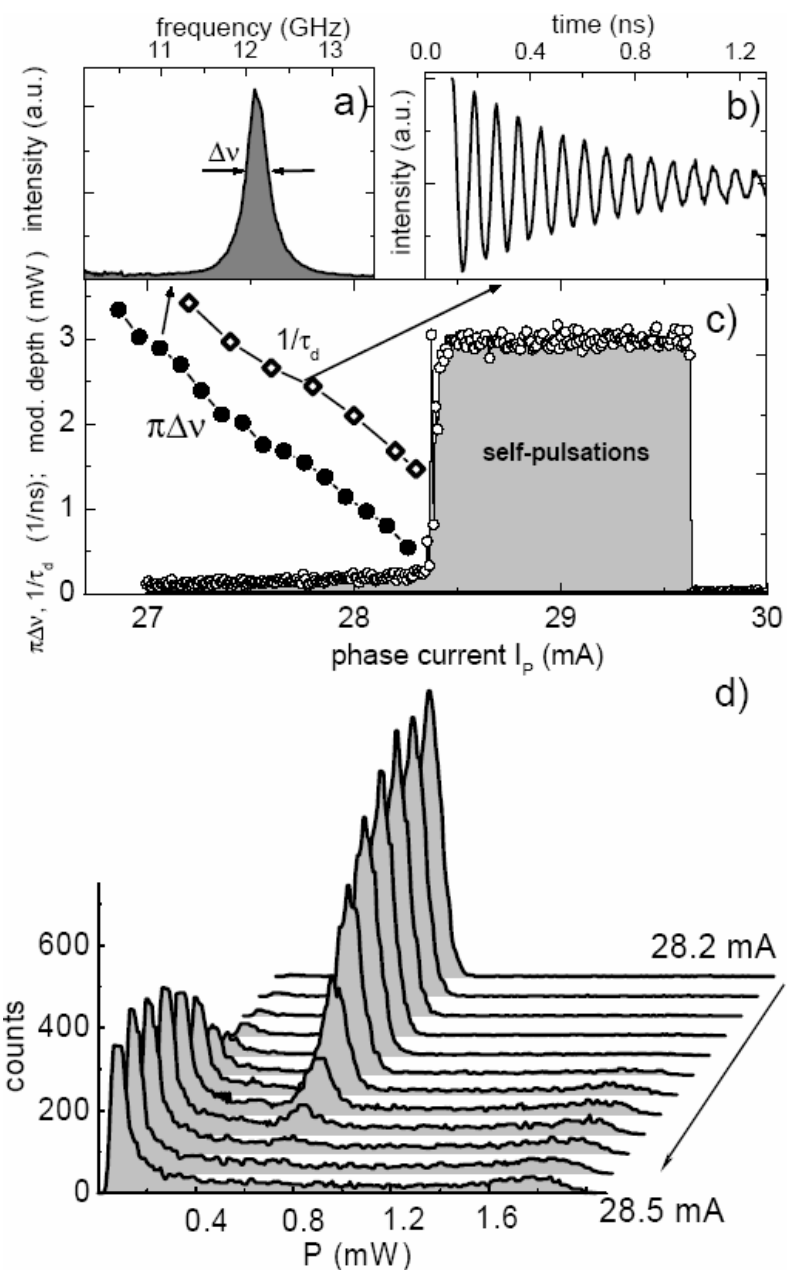

Figure 2.7: a) Characteristics of the Hopf-bifurcation of RO self-pulsations. DFB current at $50 \mathrm{~mA}$. Small circles: modulation depth. Full circles: halfwidth $\pi \Delta \nu$ of the resonance line in the rf spectrum. Open circles: damping rate $\tau_{d}^{-1}$ of relaxation oscillations. Both linewidth and decay rate approach zero. The horizontal offset of the decay rates is possibly due to a change of the point of operation caused by the external excitation. b) rf spectrum, c) time decay of relaxation oscillations, d) set of histograms with a change of the phase current.

The evolution of pulsations can also be observed in the histograms (Fig. 2.7b). The stationary state at $28.2 \mathrm{~mA}$ is represented by a single peak with about $0.8 \mathrm{~mW}$ mean power. At $28.5 \mathrm{~mA}$, the histogram shows the typical features of RO pulsations. However, this transition is not sudden as might be concluded from panel a). In a finite intermediate range, the RO-peaks appear smoothly 

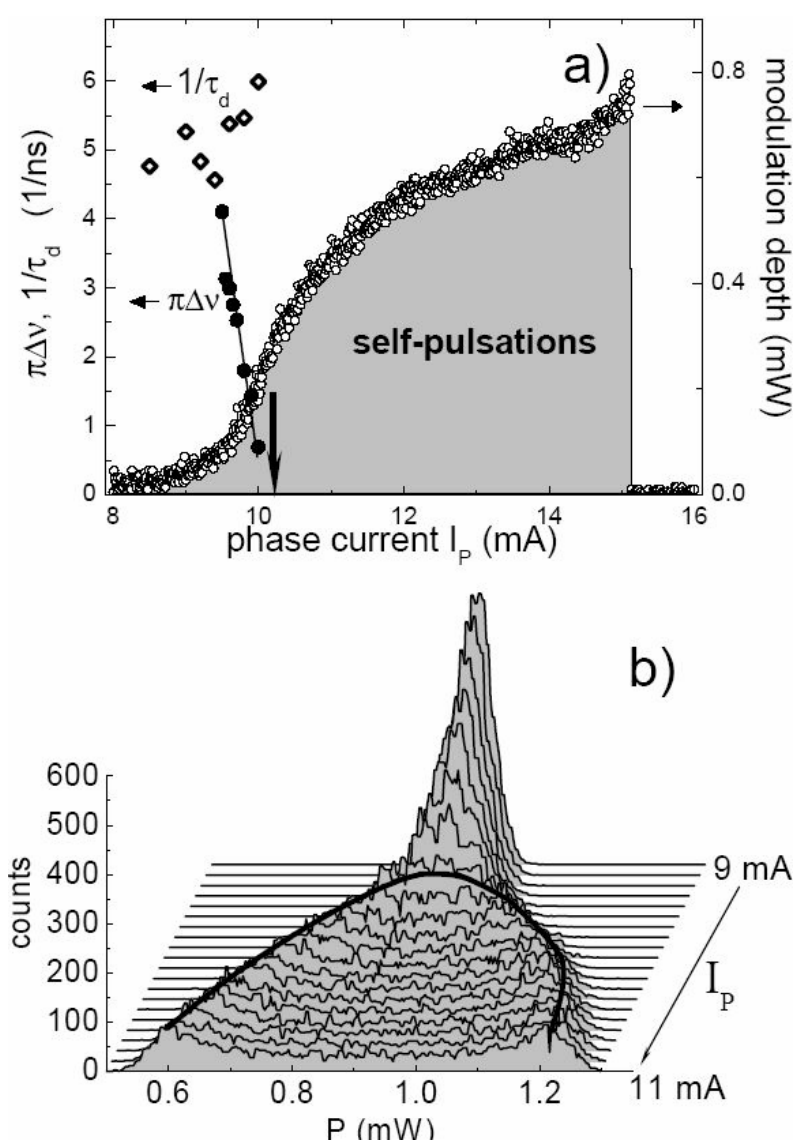

Figure 2.8: a) Characteristics of the Hopf-bifurcation of mode-beating selfpulsations. DFB current at $70 \mathrm{~mA}$ Small circles: modulation depth. Full circles: half-width $\pi \Delta \nu$ of the resonance line in the rf spectrum. Open circles: damping rate $\tau_{d}^{-1}$ of relaxation oscillations. b) Evolution of histograms with phase current, solid line to guide eyes.

in the same manner as the single cw-peak drops down. We attribute this phenomenon to noise induced transitions between the two stable attractors close to the subcritical Hopf bifurcation. Obviously, the transitions from cw to $\mathrm{RO}$ and back appear with comparable probabilities within this range. This feature confirms the proximity to a degenerate Hopf.

The same technique is applied now to the mode-beating pulsations on a horizontal cut crossing point MB in Fig. 2.5. The spectra of the SP along the cut are similar to that in point MB (Fig. 2.4). They indicate nearly sinusoidal mode-beating pulsations with frequencies around $20 \mathrm{GHz}$. The left boundary of the pulsation region depicted in Fig. 2.8 is not sharp, the modulation depth raises smoothly here. However, the mode-beating line in the power spectrum 
emerges from a precursor whose width falls rapidly when approaching the pulsation region. Thus, the mode-beating pulsations are also born in a Hopf bifurcation as predicted theoretically [ERGK00]. The measured decay rates of relaxation oscillations keep high along this bifurcation. Thus, the oscillator undergoing the Hopf bifurcation is not connected with the relaxation oscillations.

The evolution of the histograms in Fig. 2.8b exhibits similar tendencies as previously observed in the case of the subcritical Hopf bifurcation. The height of the main peak decreases and its width increases with the increase of the current. However, the two peaks of the pulsating state appear now in a smooth transitions which clearly resembles the features of a supercritical Hopf bifurcation.

\subsection{Theoretical Analysis}

The amplitude returning to the DFB at time $\mathrm{t}$ is given by the amplitude emitted a time $\tau$ before as

$$
E^{-}(t)=K e^{-i \phi} E^{+}(t-\tau) .
$$

A fraction $K$ of the field is re-injected into the laser cavity with a phase shift $\phi$ and a delay $\tau$. Let us consider stationary states. They behave as $E^{ \pm}(t)=E_{0}^{ \pm}(\Omega) e^{-i \Omega t}$, where $\Omega=\omega-\omega_{0}$ describes the deviation from the frequency of solitary laser $\omega_{0}$; The two amplitudes are also related by the reflectivity $r(\Omega)$ of the laser,

$$
E_{0}^{+}(\Omega)=r(\Omega) E_{0}^{-}(\Omega) .
$$

Substitution Eq.(2.6) into Eq.(2.7) gives $E_{0}^{+}(t)=r(\Omega) K e^{-i \phi} e^{i \Omega \tau} E_{0}^{+}(t)$, from where follows the round trip condition for the compound-cavity modes:

$$
q(\Omega, N) \exp (-i \Omega \tau)=K \exp (-i \phi),
$$

where $N$ is the average carrier density in the laser. The DFB enters here by its inverse amplitude reflectivity [CWP98]

$$
q=\frac{1}{r(\Omega)}=\frac{i \gamma}{\kappa} \cot \left(\gamma L_{D F B}\right)-\frac{\beta}{\kappa}
$$

with $\gamma^{2}=\beta^{2}-\kappa^{2}$ and the propagation constant

$$
\beta(\Omega, N)=\frac{1}{2}\left[\left(i+\alpha_{H}\right) g^{\prime}\left(N-N_{t r}\right)-\alpha_{0}\right]+\frac{\Omega}{v_{g}} .
$$


This characteristic equation allows to determine the lasing modes of the feedback setup. For moderate feedback strengths only small changes of the laser state are expected and the modes in the feedback setup are located close to the solitary positions.

The parameters involved $\left(\kappa=130 \mathrm{~cm}^{-1}\right.$ : coupling coefficient of index grating, $\alpha_{H}=-5$ : linewidth enhancement factor, $\alpha_{0}=25 \mathrm{~cm}^{-1}$ : background absorption, $g^{\prime}=10^{-20} \mathrm{~m}^{2}$ : differential gain, including the transverse confinement factor, $N_{t r}=10^{24} \mathrm{~m}^{-3}$ : carrier density at transparency level) have been deduced from independent measurements.

The solutions of the complex valued Eq. (2.8) are quite complicated surfaces in the three dimensional space spanned by $\operatorname{Re} \Omega, \operatorname{Im} \Omega$, and $N$. Fig. 2.9 depicts these solutions by curves of constant $K$ and constant $\phi$ in two different plane cuts through this space. The modes of the solitary laser $(K=0)$ are represented by vertices, where the equi-phase lines move together. The left panels shows the so-called external cavity modes. They belong to a fixed $N$, here the threshold density of the solitary laser. Obviously, the very short delay of our device has a considerable impact on the mode structure. Without delay, curves of constant feedback strength $|q(\Omega, N)|=K$ are orbits around the mode of the solitary laser. Their extension is an increasing function of $K$. With delay, the factor $\exp (i \Omega \tau)$ in Eq. 2.8 gives rise to additional modes. At small $K=0.1$, these new external cavity modes are highly damped and located on a separate line well above the orbit of the central laser mode. At the higher $K=0.3$, this line has moved down and it merged with the central orbit forming a deep valley.

Fig. 2.9c shows the central part of the same scenario in terms of modes at threshold, often called compound cavity modes. Standard single-mode stability analysis $\left[\mathrm{UBB}^{+} 04\right]$ of these modes yields two types of instabilities: saddles in the region labelled 'anti-modes' and undamped relaxation oscillations in the small island denoted by 'URO'. The grey borders of these islands represent saddle-node and Hopf bifurcations, respectively. The orbit $K=0.1$ touches the 'URO' island, but the more extended orbit $K=0.3$ does not touch the island. This island topology explains, why RO pulsations are only found in higher phase periods of $I_{P}$ (see Fig. 2.5), as $K$ is sufficiently reduced here. It is also consistent with the observation of similar Hopf bifurcations for longer cavities $\left[\mathrm{HFE}^{+} 03\right]$. Undamped relaxation oscillations are hence a common feature of short and ultra-short cavities with weak feedback.

While the form of the orbit in the right panel is independent on $\tau$, the delay controls the number of modes enclosed. For small $K, \exp (i \Omega \tau) \approx 1$ holds along the orbit. Only one mode exists that rotates with $\phi$ clockwise around the orbit. However, variation of $\Omega \tau$ along extended orbits is associated with a change of $\phi$ by more than one period, enabling thus several modes. It is this 

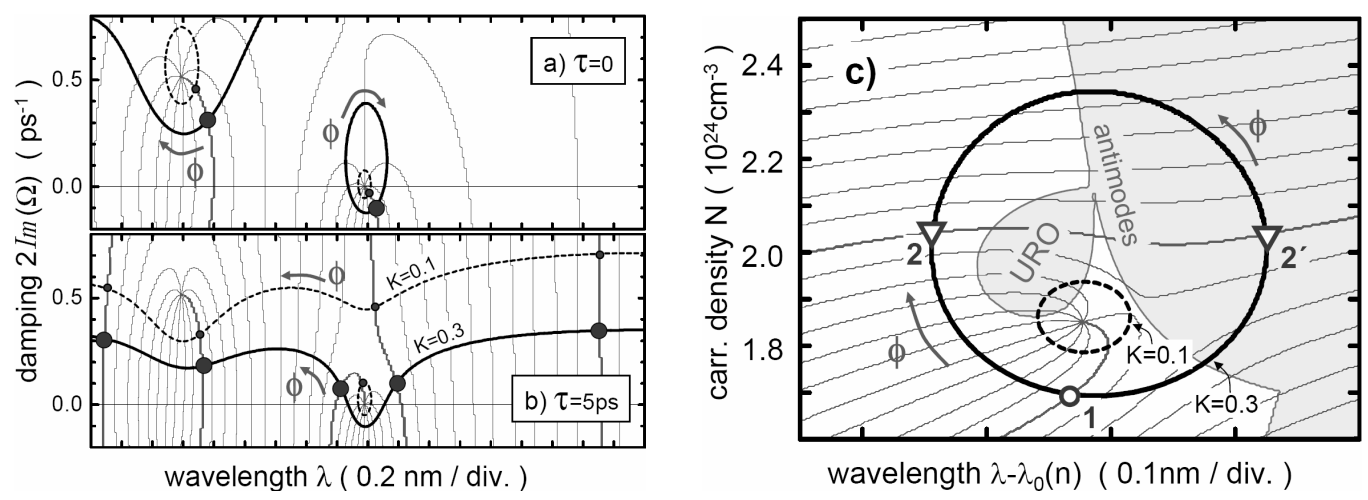

Figure 2.9: Modes calculated from Eqs. (2.8) to (2.10). Curves of constant feedback ( $K=0.1$ dashed, $K=0.3$ solid) and curves of constant phase (thin grey, 10 lines per phase period) are drawn in all panels. Panels a) and b): External cavity modes in the damping-wavelength plane, carriers fixed to the threshold density of the solitary DFB laser. Panel c): Compound cavity modes in the wavelength-density plane $\operatorname{Im}(\Omega)=0$. The carrier-induced shift $\lambda_{0}(N)$ is subtracted here for clarity. Gray area: regions of instability.

interrelation between feedback delay and strength that gives rise to new dynamics, even though $\tau / \tau_{R} \ll 1$. The variation of $\phi$ is not monotonous along the orbit. The equi-phase lines are tangential with the orbit, where it enters the anti-mode region. Pairs of modes appear or disappear here in saddlenode bifurcations. With increasing $\phi$, the stable modes move up on the left part of the loop, while the unstable anti-modes do so on the right-hand side.

At a particular phase, mode and anti-mode arrive at comparable threshold densities (triangles). Here, both modes accommodate to each other at a common density and MB pulsations emerge in a Hopf bifurcation [TP94; WT02]. This scenario is reminiscent of the bifurcation bridges predicted for long delay times [ERGK00]. The peculiar feature of the ultra-short cavity regime is the existence of only one anti-mode, yielding regular dynamics, whereas the pulse-package scenario $\left[\mathrm{HFE}^{+} 03\right]$ involves multiple anti-modes. The device output is therefore qualitatively different. Pulse packages are pulse sequences with a repetition rate exactly given by the external cavity frequency $1 / \tau$, amplitude-modulated with a frequency below $1 / \tau_{R}$. At variance, the MB pulsations are not modulated and their frequency is by about one order of magnitude smaller than $1 / \tau$, due to pulling of the side-mode by the DFB resonance. The transition between both regimes is an interesting subject of future research. We note finally that the instability above state 2 is also the source of the hysteresis observed experimentally. For decreasing $\phi$, state 1 
moves to longer wavelengths and, in contrast to forward phase, reaches the saddle-node bifurcation. Here, the laser switches to the only stable state of same $\phi$ on the left part of the orbit.

A single-mode DFB laser with an biased passive feedback cavity has been investigated experimentally. Although the 5 ps feedback delay is shorter by two orders of magnitude compared to the period of relaxation oscillations, it is crucial for the dynamics of the device. Two different types of self-sustaining intensity-pulsations are detected depending on strength and phase of the feedback which are controlled by the bias to the passive section. One type of pulsations is emerging in a Hopf-bifurcation from relaxation oscillations. These oscillations become undamped due to dispersive self-Q switching. The second type of pulsations is a mode-anti-mode beating pulsation. It is also born in a Hopf bifurcation. These findings represent experimental evidence for recent theoretical predictions [ERGK00]. A supplementary mode and stability analysis agrees well with measurements and it is used to discuss the relations to intermediate-delay $\left[\mathrm{HFE}^{+} 03\right]$ as well as zero-delay regimes.

\subsection{Period doubling}

As was shown above two types of pulsations can emerge in a laser with optical feedback. A Hopf bifurcation is responsible for their appearance. However, transition to higher order instabilities is observed in such a type of lasers, e.g., chaos $\left[\mathrm{HFE}^{+} 03\right]$. In this section we consider a period doubling (PD) bifurcation.

A sequence of power spectra and histograms is shown in Fig.2.10 for the evolution of the dynamical state with change of phase current $I_{P H}$. The power spectrum with a frequency of $12 \mathrm{GHz}$ and distinct harmonics at $24 \mathrm{GHz}$ and $36 \mathrm{GHz}$ in Fig.2.10 (a1) corresponds to URO pulsations being appeared in a subcritical Hopf bifurcation. A histogram of this state is shown in Fig.2.10 (a2) where two spikes feature is attributed with periodic pulsations. Suddenly, after increasing the phase current by a few tenth of milliamperes, a single small peak at a half of the fundamental frequency $f_{0} / 2$ appears, shown in Fig.2.10 (b1). In the histogram, the sharp spikes become lower and broader, see panel (b2). The low frequency peak $f_{0} / 2$ further increases in amplitude and narrowed in frequency width; its harmonics are clearly visible Fig.2.10 (c1). Peaks in the histogram begin to split up panel (c2), indicating that the period doubling is due to an amplitude modulation with frequency $f_{0} / 2$. A well developed period doubled state is represented by the power spectrum in Fig.2.10 (d1). A small peak at $3 f_{0} / 4$ and its harmonics are seen as well. These peaks correspond to a precursor of a second PD. 
Further increasing of the phase current leads to sudden disappearance of the oscillatory state. In the power spectrum a flat noisy background appears. In the histogram a single spike corresponds to stationary emission (cw state).

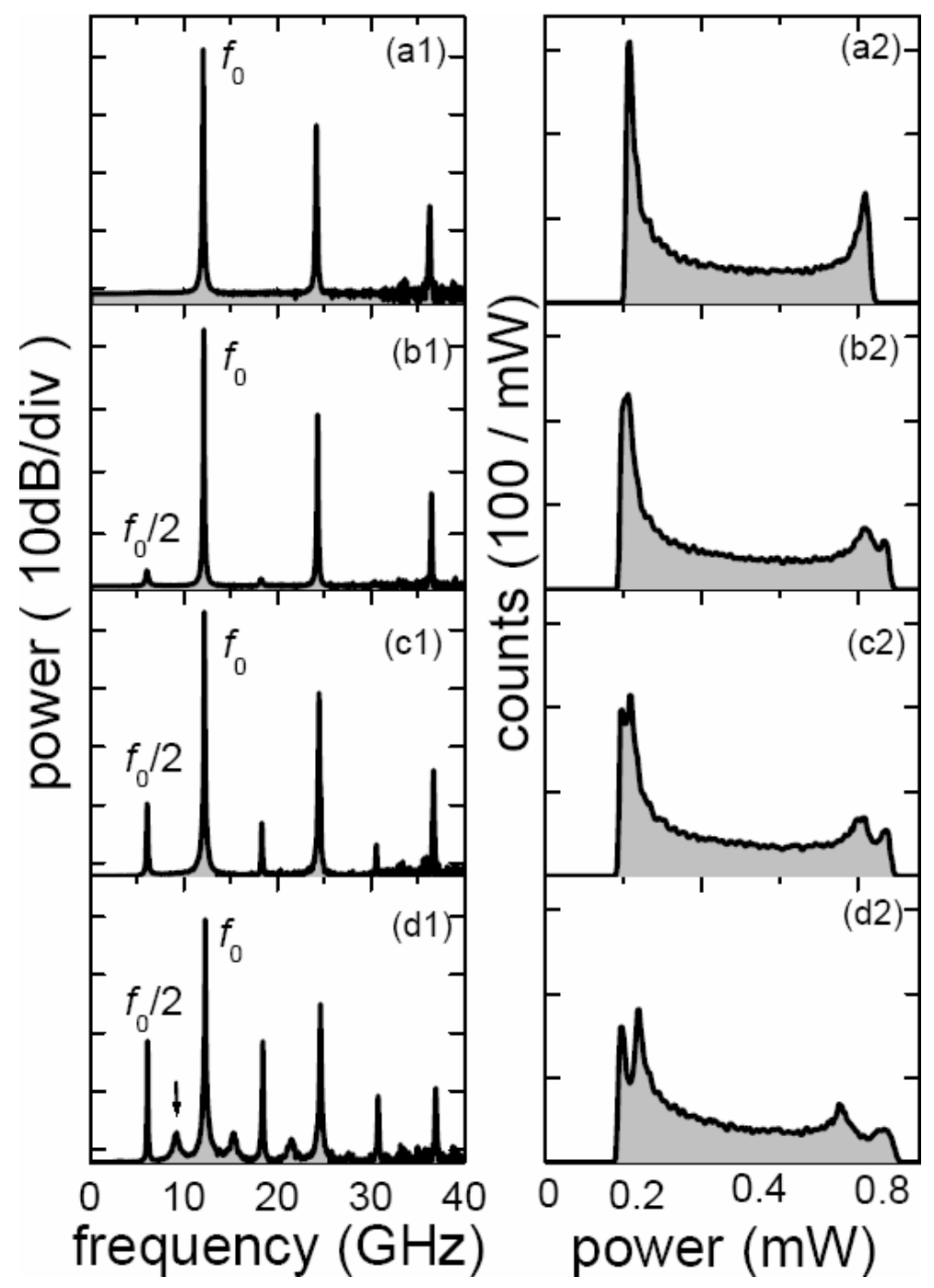

Figure 2.10: Power spectra (left panel) and histograms for corresponding states (right panel). Current in DFB section is constant $I_{D F B}=90 \mathrm{~mA}$. From (a) to (d) phase current $I_{P H}$ takes the values $18.6 \mathrm{~mA}, 19 \mathrm{~mA}, 19.2 \mathrm{~mA}, 20$ $\mathrm{mA}$. An arrow indicates the peak at the frequency $3 f_{0} / 4$

The sequence of period doubling bifurcations is a typical route to chaos. We have observed only the first two PD bifurcations in the sequence. Probable explanation of this phenomenon is that the attractor after two PD bifurcations collides with a saddle in a homoclinic bifurcation and disappears 
[WKL01]. Emergence of chaotic motion in semiconductor laser with optical feedback depends of feedback strength [Kra00].

In order to obtain chaotic motion one needs to increase feedback strength. This was performed in the laser with active optical feedback. Dynamics of this laser is studied in the next chapter. 


\section{Chapter 3}

\section{Chaotic motion}

So far we considered MB and URO pulsations separately. In this section we investigate the nonlinear interaction of these two type of pulsation. The mixed state results in motion on a torus in phase space. Fractalization of the torus leads to chaotic motion. Experimental measurements are supplemented by simulations of a comprehensive traveling-wave equations (TWE).

\subsection{Quasi-periodic route to chaos}

Figure 3.1 sketches an amplified feedback laser (AFL). The basic setup is similar to the passive feedback laser, Sec. 2.2, however, the feedback cavity is implemented as a combination of passive and amplifier section. This allows for a separate control of feedback phase (phase section) and feedback strength (amplifier section) using current injection. Strong feedback is adjustable since the amplifier can compensate for losses arising in the device (i.e. in the phase tuning section, at the section interfaces, and the amplifier facet). Moreover, the gain in the amplifier section can increase the overall internal photon density, producing new effects in comparison to passive feedback. The dynamics of the additional carrier density in the amplifier will turn out as important for the device dynamics. The dynamics of AFL is investigated in Ref. $\left[\mathrm{BBK}^{+} 04 ; \mathrm{Bau} 05\right]$. It has been shown that after sequence of bifurcations laser output may become chaotic. However, the investigation of this chaotic motion was not performed in detail. Here, we analyze in depth the sequence of bifurcations which leads to chaotic motion.

The experimental setup is shown in Fig.3.1. The power spectra are measured by a power spectrum analyzer (R\&S FSP 9) with $40 \mathrm{GHz}$ bandwidth. Analysis of temporal characteristics is performed by a digitizing sampling oscilloscope (HP 54120B) with bandwidth of $50 \mathrm{GHz}$. Ultra fast photodi- 


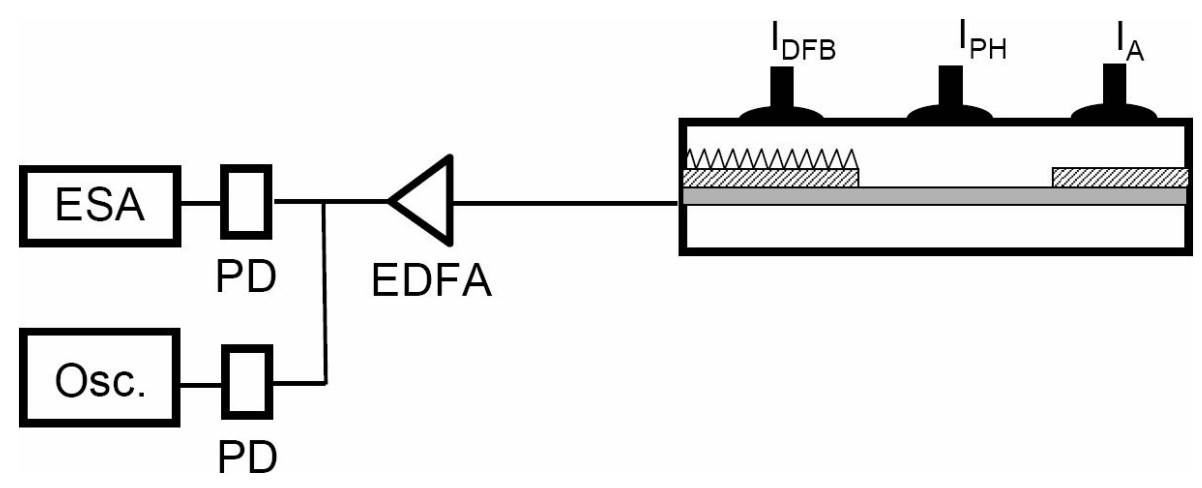

Figure 3.1: Schematic of an AFL. An additional amplifier section enables the control of feedback strength. The different sections are controlled by independent currents. ESA - power spectrum analyzer, EDFA - erbium doped fiber amplifier, Osc - digitizing sampling oscilloscope.

odes $\left(u^{2} t\right)$ are used as a detectors. Three currents govern the dynamics of the device $I_{D F B}$ - laser current, $I_{P H}$ - phase current and $I_{A}$ - current in the amplifier section. We fixed two currents: $I_{D F B}=41.7 \mathrm{~mA}, I_{A}=47 \mathrm{~mA}$. The phase current is used as a bifurcation parameter as well as in Sec.2.4. Sampling techniques allow to analyze ultra-fast periodic processes, but the analysis of quasi-periodic and chaotic processes by this technique is a challenge. To acquire information about the form of time traces we supplemented our measurements with a histogram.

Fig.3.2 (upper panel) depicts a bifurcation diagram, maxima and minima of the laser power versus a phase current $I_{P H}$. The panels below show power spectrum and histogram in different operation points. The laser operates in $\mathrm{cw}$ regime at low phase currents. In the power spectrum this state is presented by a flat noisy background (a1). A small peak at $3 \mathrm{GHz}$ corresponds to noise induced damped relaxation oscillations. In the histogram one observes a sharp peak at the mean power (a2). In the power spectrum a spike appears at $34 \mathrm{GHz}$ what is typical for mode beating pulsations. The histogram exhibits two spikes feature of sine-wave oscillations. The amplitude of pulsations increases as a square root with the phase current $A \sim\left(I-I_{c}\right)^{1 / 2}$ what indicates a supercritical Hopf bifurcation, see Fig.3.2 (upper panel). The amplitude of pulsations start to saturate at currents above $5 \mathrm{~mA}$.

Suddenly at $6.8 \mathrm{~mA}$, the amplitude begins to grow again. In the power spectrum a peak at $4 \mathrm{GHz}$ appears, (c1). This indicates a secondary Hopf or a torus bifurcation. Behind the torus bifurcation, motion with two frequencies occurs. The trajectory wanders on a torus in the phase space. The origin of the newly born pulsations is undamped relaxation oscillations (URO). 

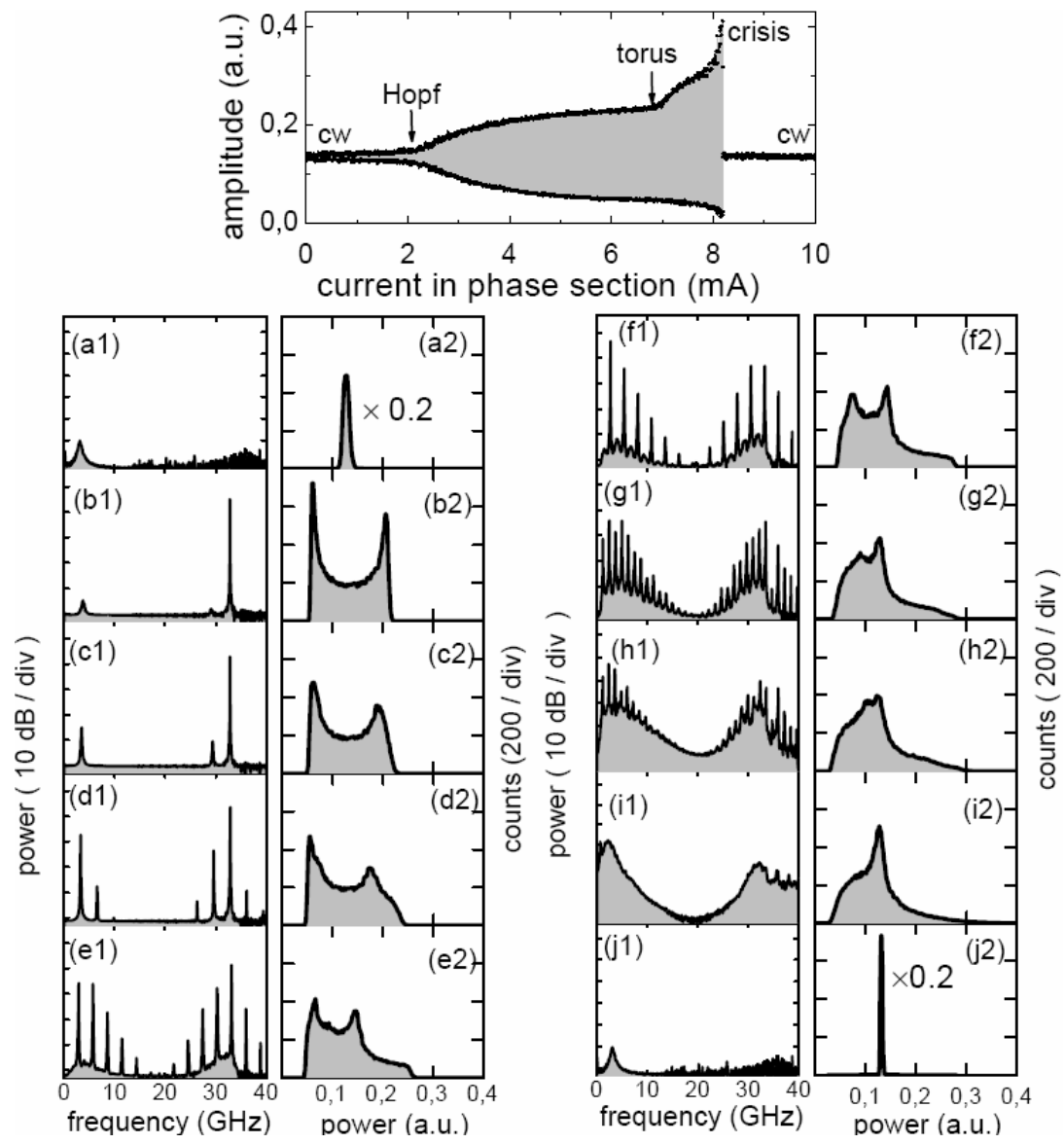

Figure 3.2: Upper panel: maxima and minima of laser intensity vs. current in the phase section $I_{P H}$. Evolution of states, first and third columns: power spectra, second and fourth column: probability distribution function - histogram. From (a) to $(\mathrm{j}) I_{P H}$ takes values $1.5 \mathrm{~mA}, 6 \mathrm{~mA}, 6.8 \mathrm{~mA}, 7 \mathrm{~mA}, 7.3$ $\mathrm{mA}, 7.5 \mathrm{~mA}, 7.7 \mathrm{~mA}, 7.9 \mathrm{~mA}, 8.05 \mathrm{~mA}, 8.2 \mathrm{~mA}$.

Further increasing of phase current leads to a growth of the amplitude of the low frequency pulsations. In the histogram one observes a bump at high power, see $(\mathrm{d} 1)$. The power spectrum exhibits a new peak at $8 \mathrm{GHz}$ what corresponds to higher harmonics of URO pulsations. The peaks at 34-4 $\mathrm{GHz}, 34-8 \mathrm{GHz}$ are due to nonlinear interaction of URO and MB pulsations.

At $7.3 \mathrm{~mA}$ one observes a comb of peaks in the power spectrum what corresponds to a well developed torus (e1). The histogram shows that the bump at the high power becomes flatter and spikes attributed with periodic motion approaching to each other and becomes smaller (e2).

A broadband feature emerges in the power spectrum as the phase current 
is increased (f1). The histogram shows that the bump at the high power becomes more flatter and spreads towards high power (f2). Peaks of periodic motion become less resolvable.

At $7.7 \mathrm{~mA}$, a peak at half of the low frequency $(2 \mathrm{GHz})$ appears. This may indicate a period doubling bifurcation of the torus. But also it may point out a complex resonance on the torus[WKL01]. Resonances on the torus occur when ratio of torus frequencies takes a rational value. Resonance on the torus in AFL is investigated in details in Bauer's thesis [Bau05] and we will not consider them.

At $7.9 \mathrm{~mA}$, an abrupt increase of the pulsation amplitude occurs. In the power spectrum one observes a broadband feature indicating chaotic motion (h1). In the histogram one observes no any marks of periodic features, a long tail decaying till high power and a bump at low power. However, spikes in power spectrum can be still visible what hints on weakly unstable periodic orbits in the CA, which are often visited.

At $8.05 \mathrm{~mA}$ the power spectrum possesses a noise like broad spectrum where the bimodal shape is reminiscent of the torus. In the histogram, one observes a unimodal distribution what is typical for chaotic vibrations. A long exponentially decaying tail is a feature of high intensity narrow spikes. The bump at low power characterizes dropouts of the laser intensity which resemble low-frequency fluctuations (LFF) in the laser with short optical feedback [San94; FTL ${ }^{+}$96]. The sharp peak in the distribution in the middle corresponds to a slowing down of the motion in this region. Two objects may induce such slowing down in phase space: a stable or a saddle equilibria. Further, we will show that the saddle is responsible for this slowing down.

Finally, as pictured in Fig. (j1), the dynamical scenario concludes in a sudden disappearance of the chaotic state, which is then replaced by stable emission. This transition depicted in Figs. (i1) and (j1) is not only sudden but also displays hysteresis. In Chapter 4 I will give experimental evidence that this sadden break down of chaos represents as boundary crisis. It is structurally stable, i.e., it appears on extended line in the parameter space spanned by the currents $I_{D F B}, I_{P H}, I_{A}$.

In the following sections, we will confirm by numerical investigation the dynamics underlying the transitions over one cycle of the feedback phase depicted in Fig. 3.2.

\subsection{Numerical experiment}

We supplement the analysis of experimental data by performing simulations with the comprehensive traveling wave equations (TWE) model solved by the 
software suite LDSL (Longitudinal Dynamics in multisection Semiconductor Lasers) of the Weierstrass Institute ${ }^{1}$. Parameters and details of the model can be found in Appendix B and Appendix C.

In Fig. 3.3, we show the calculated maxima of the output power as a function of the feedback phase $\phi$. We start from the situation that laser

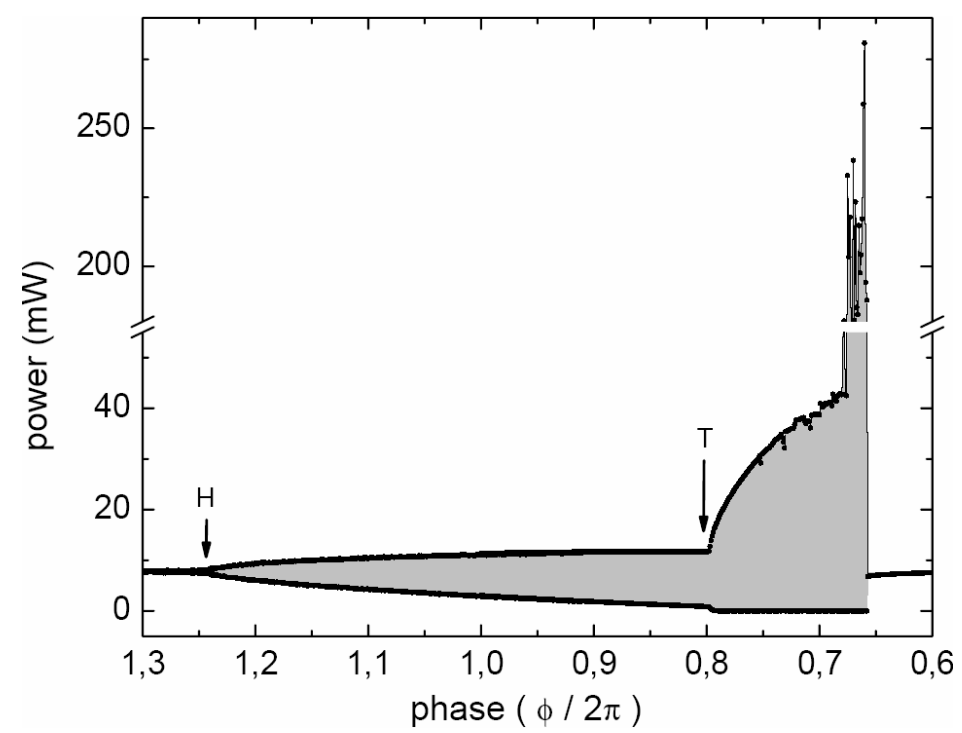

Figure 3.3: Maxima and minima of output power versus phase $\phi . \mathrm{H}$ - Hopf bifurcation, $\mathrm{T}$ - torus bifurcation.

emits constant intensity (stable stationary state). As $\phi$ is decreased, a Hopf bifurcation takes place and the laser now produces periodic output (Fig. 3.4(a)). Sine form oscillations with period 25 ps originates from MB pulsations (Fig.3.4 (a2)). In phase space projection (optical power versus carrier density in DFB section) a closed loop characterizes the limit cycle. When increasing $\phi$ further, a torus bifurcation (or Neimark-Sacker bifurcation) takes place, so that, after transients all trajectories settle down to an attracting torus, corresponding to quasiperiodic (or very high-period locked) dynamics, Fig. 3.4(b). The power spectrum shows a new spike at $4 \mathrm{GHz}$, see Fig.3.4 (b3), what is typical for URO pulsations. In time series one observes that fast oscillations are modulated by low frequency pulsations. Upon increasing $\phi$ further, the torus starts to breakup into a chaotic attractor (Fig. 3.4(c)) . For certain ranges of $\phi$, the dynamics on the breaking-up torus may be locked to lower-period periodic orbits (Fig. 3.4(c3)). At 0.688 value of phase parameter a sudden increase in the size of the attractor occurs due to a global

\footnotetext{
${ }^{1}$ http://www.wias-berlin.de/software/ldsl/
} 


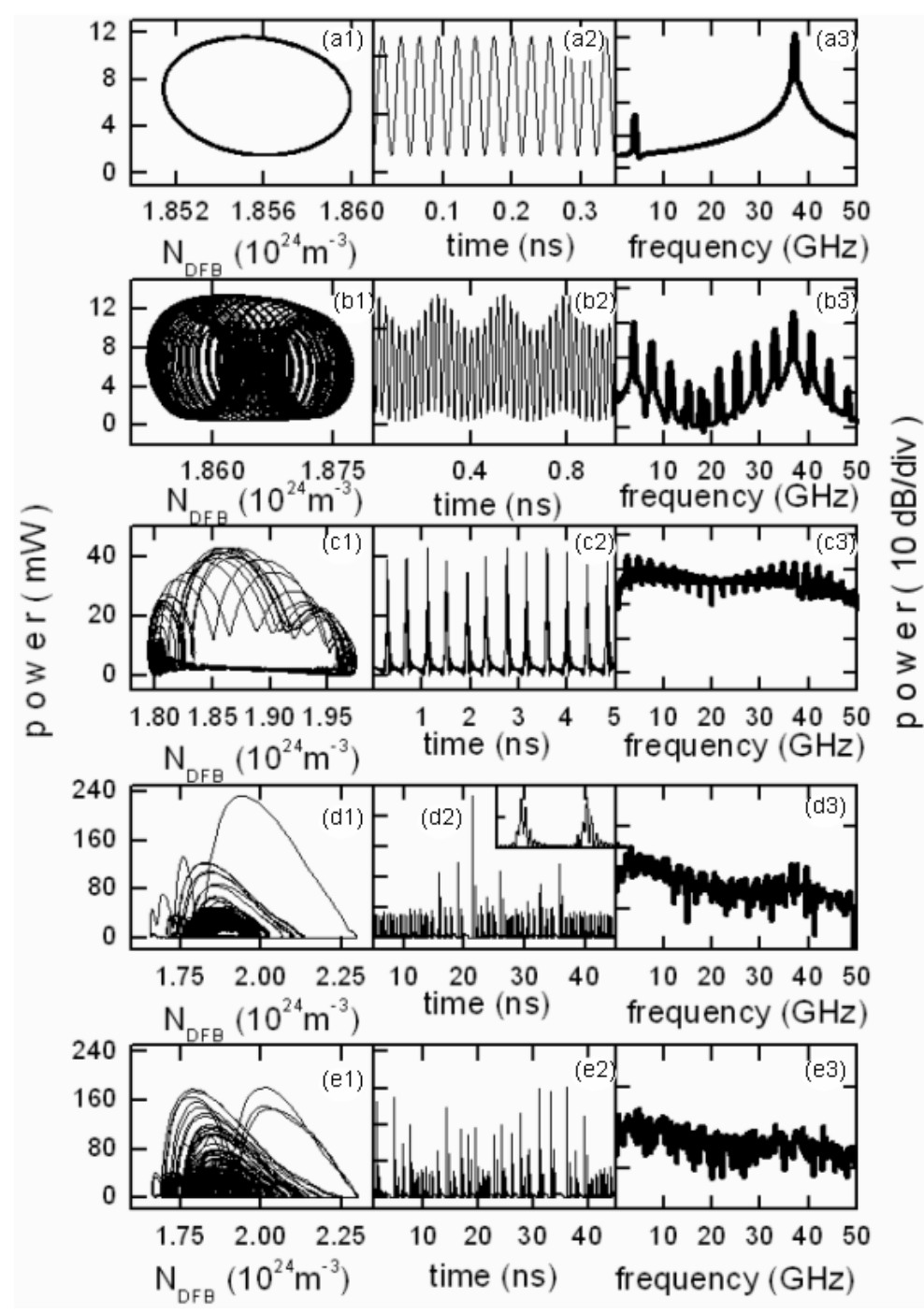

Figure 3.4: Changes of the dynamics when the phase $\phi$ varies, $I_{D F B}$ and $I_{A}$ are constant and equal $41.7 \mathrm{~mA}$ and $49.5 \mathrm{~mA}$, respectively. Shown are the phase space projections in the plane of power from left facet vs $n_{D F B}$ (first column), the time trace (second column), the power spectrum (third column). From (a) to (e) $\phi / 2 \pi$ takes the values $0.88,0.8,0.7,0.688,0.66$. Note the different scales.

bifurcation (Fig. 3.3). The localized chaotic attractor hits the stable manifold of the saddle immediately above it. After this bifurcation, the dynamics can leave the previous region of localization to make a large excursion ending with a global reinjection. This global bifurcation is, hence, identified as an interior crisis [GOY82]; the size (and nature) of the attractor suddenly 
changes. The chaotic attractor of Fig. 3.4(c) collides with a saddle, resulting in the much larger chaotic attractor in Fig. 3.4(d). The saddle is now part of the new attractor. An important ingredient of this bifurcation is the presence of the reinjection mechanism. Without reinjection the dynamics would never return to the region of the former attractor. After the interior crisis, the dynamics is of a typical form and it displays intermittency because the dynamics stays near the previous localized attractor for long periods of time interrupted by sudden large excursions (compare Fig. 3.4(d1) and (e1)).

As $\phi$ is increased away from the interior crisis, these large excursions become more frequent until the dynamics moves more uniformly over this large new chaotic attractor (Fig. 3.4(e2)). Eventually the chaotic attractor disappears suddenly in a second global bifurcation. This bifurcation is a boundary crisis [GOY82], in which the attractor hits its basin boundary and suddenly disappears, see Fig.3.3. Indeed, we found the typical long chaotic transients associated with this bifurcation before the system settles down to the stationary state. Properties of the chaotic transients will be further addressed in Chapter 4.

The numerical analysis enables us to quantify the chaotic dynamics by computing the Lyapunov exponents. For this goal, the partial-differential TW equations are approximated by ordinary differential equations (ODE) for a truncated set of optical modes [Rad06]. The number of modes is carefully chosen to reproduce the solutions of the full TW model in the relevant $\phi$ range. The Lyapunov exponents of the resulting 17 real ODE are calculated as described in Ref. [WSSV85]. The Lyapunov spectra are nearly independent of $\phi$. In decreasing order, the first four exponents have approximate values of 2.7, $0,-1.0$, and $-3.0 \mathrm{~ns}^{-1}$. The respective Kaplan-Yorke dimension varies between 3.3 and 3.7 and is thus indicative of a high-dimensional $\mathrm{CA}$. The rotational invariance of the optical equations is not accounted here. It gives another zero Lyapunov exponent and increases the attractor dimension by 1 .

Comparing of experimental and numerically calculated data one observes good agreement in dynamical behavior. In the experiment and as well as in the calculations, stationary state loose its stability in a supercritical Hopf bifurcation where MB pulsations appear. A supercritical secondary Hopf or a torus bifurcation leads to motion on the torus in phase space. The power spectrum of this trajectory matches very well to the one shown in Fig. 3.2 (b3). Deformation of the torus in phase space results in short narrow pulses separated by intervals with almost constant power (compare Fig.3.4(c2) and Fig.3.2(g2)). Sudden change of the quasiperiodic attractor can be seen in the experiment (Fig.3.2(i)) and in the calculation (Fig.3.4(d)) due to internal crisis. Boundary crisis is responsible for abrupt disappearance 
of the chaotic vibration. In Chapter 4 dynamics close to the boundary crisis will be investigated in detail.

\subsection{Conclusion}

We have provided a detailed overview over the dynamics of the laser with active optical feedback. When changing $\phi$ phase over $2 \pi$, we observed experimentally a transition from stable output via periodic and quasiperiodic output to chaotic motion and back to stable output.

To analyze these experimental observations, the TWE model equations are solved numerically. It has been shown that two global bifurcations are involved in dynamics of the laser with ultrashort optical feedback. Internal crisis is responsible for explosion of chaotic attractor and boundary crisis for its disappearance. 


\section{Chapter 4}

\section{Excitability of Chaotic Transients}

In this chapter I give experimental evidence that the sudden destruction of chaos, described in Chapter 3, is occurred due to a boundary crisis. The existence of high-dimensional chaotic transients (CT), which are a fingerprint of the boundary crisis, is demonstrated. In numerics, CT are usually detected when switching the parameter across the transition. This is impossible in experiment due to extremely short time scales. Instead, the CT are excited by short optical pulses. In course of these experiments excitability was discovered. Thus, this chapter combines two nonlinear effects, which so far are treated only separately.

The generic phase-space portrait behind our observation consists in a boundary crisis of a chaotic attractor with a saddle born in a saddle-node bifurcation of continuous-wave states. The excitation of the CT exhibits a distinct threshold as well as a refractory time.

\subsection{Chaos in crisis}

Crises are a common manifestation of chaotic dynamics for dissipative systems and have been seen in many experimental and numerical studies [GOY86; GOY82; PDEPH88; JFT94]. In a crisis, one observes a sudden discontinuous change in a chaotic attractor as a system parameter is varied. The crises are typically of three types: in the first, internal crisis, the size of the chaotic attractor in phase space suddenly increases; in the second, mergin crisis, (which can occur in systems with symmetries) two or more chaotic attractors merge to form one chaotic attractor; in the third, boundary crisis, type a chaotic attractor is suddenly destroyed as the parameter passes through its 
critical value

After a boundary crisis (BC) chaotic attractor is replaced by a nonattracting chaotic saddles (CS). A chaotic saddle is a bounded set, and it has fractal structures in both stable and unstable directions, in contrast to a chaotic attractor which exhibits a fractal structure only in the stable direction. Due to the fractal structure in the unstable direction, an infinite number of gaps of all sizes exists along the unstable manifold of the chaotic saddle. Let us consider trajectories with initial conditions in the region of the basin of attraction of the attractor which existed before crisis. Such trajectory will typically behave as a chaotic transient (CT). That is, they are initially attracted to the phase-space region formerly occupied by the attractor; they then bounce around in this region in a chaotic way; finally, after behaving in this fashion for a arbitrary long time, they suddenly move away from the region of the former attractor and asymptote some other attractor.

The length of time an orbit spends in the vicinity of the CS depends sensitively on its initial condition, but, nevertheless, when many such orbits are considered, the length of the CT apparently has a well-defined average which tends to infinity as $p$ approaches $p_{c}$. For example, let $M(t)$ denote the number of trajectories staying still inside the CS after time $t$, and take $M_{0}$ initial conditions so large that $M(t) \gg 1$. As $t$ becomes large, one observes, in general, an exponential decay in the number of survivors, that is, one finds asymptotically [GOY82] that

$$
\frac{M(t)}{M_{0}} \propto e^{-t / \tau}
$$

where $\tau$ is the average lifetime of the chaotic transient. It is well established both theoretically [GOY86; GORY87] and experimentally [DRC ${ }^{+} 89$; $\left.\mathrm{SDG}^{+} 91\right]$ that the average lifetime $\tau$ scales with the parameter variation as:

$$
\tau \propto\left(p-p_{c}\right)^{-\gamma}
$$

where $p$ is the control parameter of the system, and $p_{c}$ critical value of $p$ at the crisis. Grebogi, Ott, and Yorke proved that [GORY87], in an everywhere smooth and "exactly dissipative" mapping system, the scaling exponent should take a value of $\gamma=1 / 2$ in a one dimensional case, and $1 / 2 \leq \gamma \leq 3 / 2$ in a two-dimensional case.

\subsection{Excitability}

Excitability is observed in a wide range of natural systems. A list of examples includes lasers, chemical reactions, ion channels, neural systems, cardiovascular tissues and climate dynamics, to mention only the most important fields 


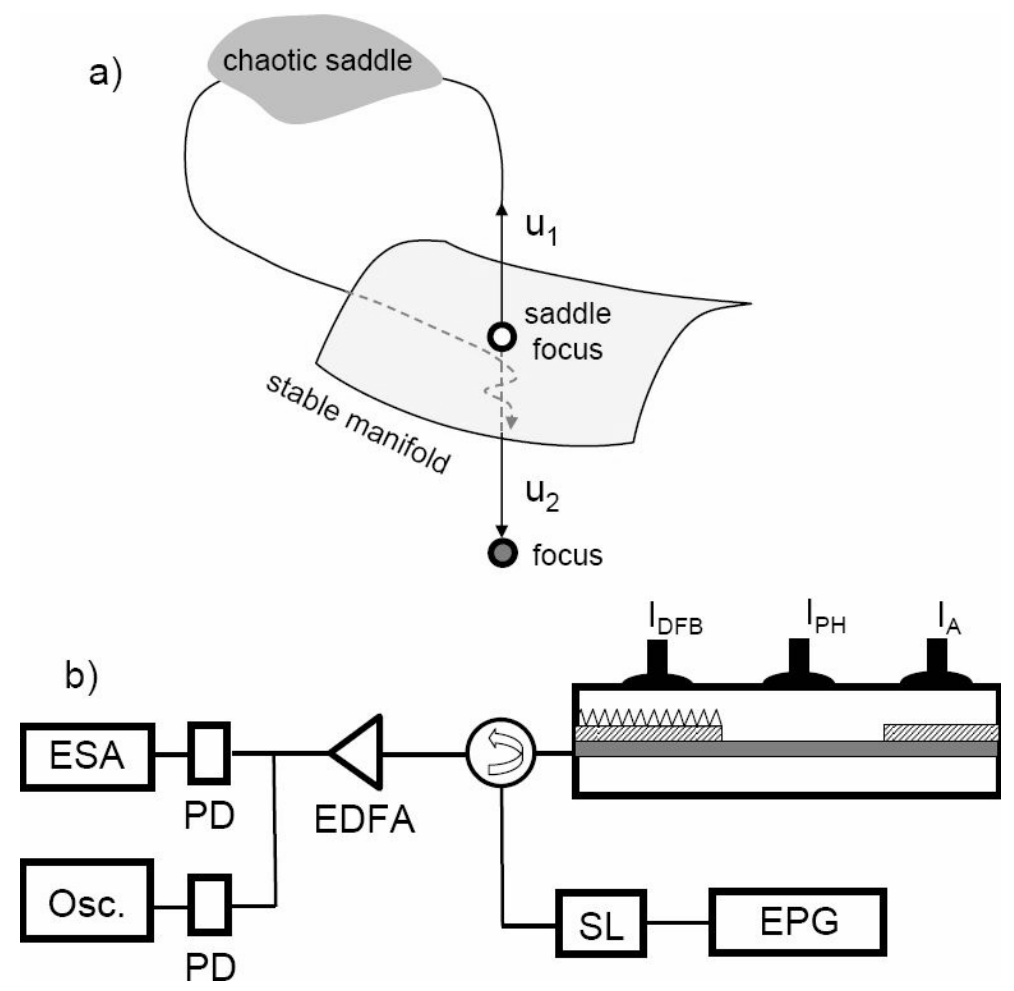

Figure 4.1: a) Schematic phase-space portrait of an excitable chaotic saddle. $u_{1}, u_{2}$ - the two branches of unstable manifold of the saddle-focus. b) Laser with external excitation. ESA - electrical spectrum analyzer, EPG electrical pulse generator, PD - photo diode, EDFA - Erbium doped fiber amplifier, SL - gain-switched DFB laser.

of research [WBRH02; Hod47; Mur93; CB99; ZZ70]. Common to all excitable systems is the existence of a "rest" state, an "excited" ("firing") state, and a "refractory" (or "recovery") state. If unperturbed, the system resides in the rest state; small perturbations result only in a small-amplitude linear response of the system. For a sufficiently strong perturbation, however, the system can leave the rest state, going through the firing and refractory states before it comes back to rest again. This response is strongly nonlinear and accompanied by a large excursion of the system's variables through phase space, which corresponds to a spike. The system is refractory after such a spike, which means that it takes a certain recovery time before another excitation can evoke a second spike. Excitability requires a certain phase space configuration. An equilibrium state (or a small-amplitude orbit) is the only attractor available and serves as rest state. Close to it, a sharp separatrix between trajectories of different types must exist. One type of 
trajectories approaches the rest state directly and determines the subthreshold response. The trajectories beyond the separatrix undertake extended phase space excursions before returning and govern the superthreshold response. Such configurations appear generically after bifurcations at which an extended periodic orbit is suddenly destructed [Izh00]. Paradigmatic examples are the Canard transition in the FitzHugh-Nagumo model [Mur93] and homoclinic bifurcations $\left[\mathrm{KSS}^{+} 03\right.$; WBRH02]. The ruin of the destructed orbit guides the long excursions after superthreshold stimulation in either case.

Our idea is to replace the regular orbit by a chaotic attractor (CA). Specifically, we consider the configuration sketched in Fig.4.1. A saddle-focus and a stable focus stemming from a nearby saddle-node (SN) bifurcation exist in close neighborhood. Branch $u_{2}$ of the unstable manifold of the saddlefocus goes to the stable focus representing the rest state. The other branch $u_{1}$ leads to a CS formed just before in a boundary crisis when a CA has touched the stable manifold of the same saddle-focus. Obviously, the stable manifold of the saddle-focus separates two completely different types of trajectories. Those below this separatrix are spiraling along unstable branch $u_{2}$ directly to the rest state. Those above follow unstable branch $u_{1}$ into the CS and reach the rest state only after moving for some time along the saddle. Accordingly, the perturbed system may exhibit two qualitatively different responses: immediate return to the rest state upon weak perturbations and a long chaotic excursion along the CS when the stimulus is strong enough to push the system beyond the separatrix. The phase-space configuration of Fig.4.1a is prepared by means of the laser with active optical feedback (AFL), see Chapter 3. The experimental setup is shown in Fig.4.1b. The response to external stimuli is studied by injecting short optical pulses into the DFB section. The pulses with a duration about $30 \mathrm{ps}$, are generated with a gain-switched DFB laser (Alcatel A1905 LMI) and electrical pulse generator (HP 8131A). The wavelength of $1530 \mathrm{~nm}$ is non-resonant to the AFL and the repetition rate is $5 \mathrm{MHz}$. The pulse energy is controlled by an optical attenuator (SK 48AT). The maximal attained pulse energy is about 10pJ. The output is analyzed by a spectrum analyzer with bandwidth 40 $\mathrm{GHz}$ or, in order to record time transients, by a digital sampling oscilloscope (HP 54120B) with bandwidth $50 \mathrm{GHz}$ and sampling rate $500 \mathrm{kHz}$.

Locating the device relatively far above the critical current in the $\mathrm{cw}$ regime, the response on optical injection is indeed very similar to excitability.

A distinct spike or set of spikes occur in the output followed by relaxation oscillations and return to stable emission within about $2 \mathrm{~ns}$. When plotting the height of the largest spike versus the energy of the stimulation pulse, an "all-or-none" response with a sharp threshold is found (Fig. 4.3). Even 

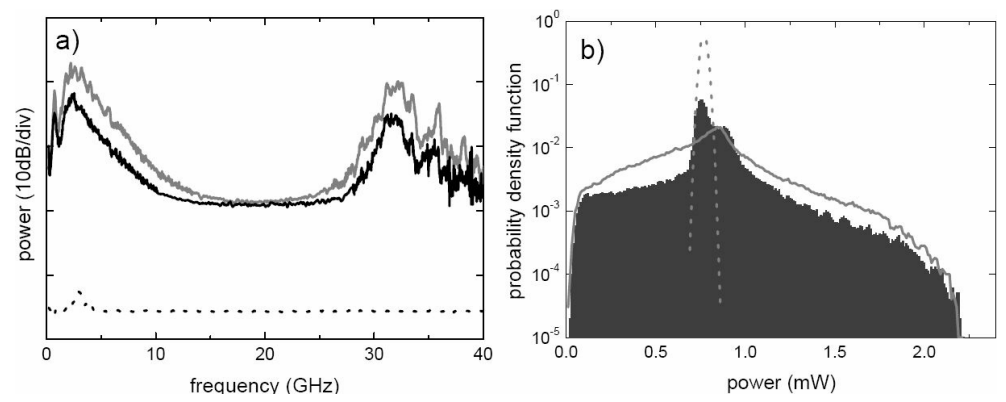

Figure 4.2: Left panel: power spectra, right panel histograms at selected phase currents, chaos $I_{P H}=6.85 \mathrm{~mA}$ (solid gray lines), stationary cw state $I_{P H}=6.9 \mathrm{~mA}$ power spectrum (dotted), histogram (grey dotted). Power spectrum (black solid line) and histogram (dark grey area) at $I_{P H}=6.9 \mathrm{~mA}$ under the superthreshold stimulation. Note the logarithmic scale in both plots.

more, using a pair of stimulation pulses, the existence of a refractory period is evidenced, see Ref. [Bet03]. The device fires only again, when the delay of the second pulse is longer than the time needed to generate the response on the first pulse. However, marked differences to the standard excitability scenario turn out, when the operation point is moved closer to the chaotic regime, as will be addressed below.

Numerical calculations based on the traveling-wave (TW) equations entirely confirm the experimentally observed pathway to chaos and reveal the underlying bifurcations. For a detailed description of the TW model as well as the parameter set, see Appendix B. In the calculations, instead of $I_{P}$, the feedback phase $\phi$ produced per round-trip in the passive section is directly used as control parameter. Fig. 4.4 compares theoretical and experimental

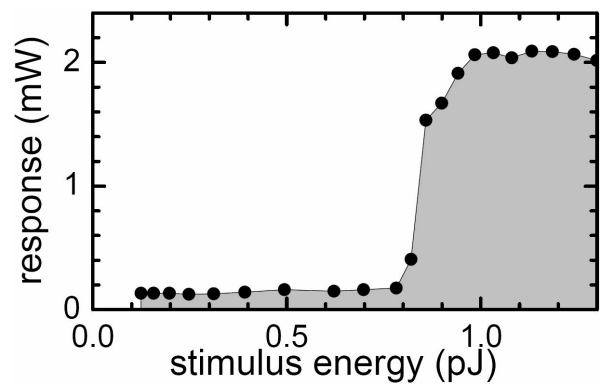

Figure 4.3: Response of the multi-section laser on 30 ps optical excitation. Maximum device output versus optical pulse energy $\left(I_{P}=6.9 \mathrm{~mA}\right)$. 
results. Note that increasing $I_{P}$ means decreasing $\phi\left[\mathrm{BBK}^{+} 04\right]$. A SN bifurcation $(\odot$ in Fig. $4.4 \mathrm{a})$ occurs still within the chaotic domain. The resultant $\mathrm{cw}$ states are the stable focus and saddle-focus required in the phase-space portrait of Fig. 4.4a. Slightly right of the SN bifurcation at critical phase $\phi_{c}$, the $\mathrm{CA}$ responsible for the chaotic behavior breaks up in a boundary crisis by touching the stable manifold of the saddle-focus. A close neighborhood of both transitions is achieved by proper choice of DFB and amplifier current.
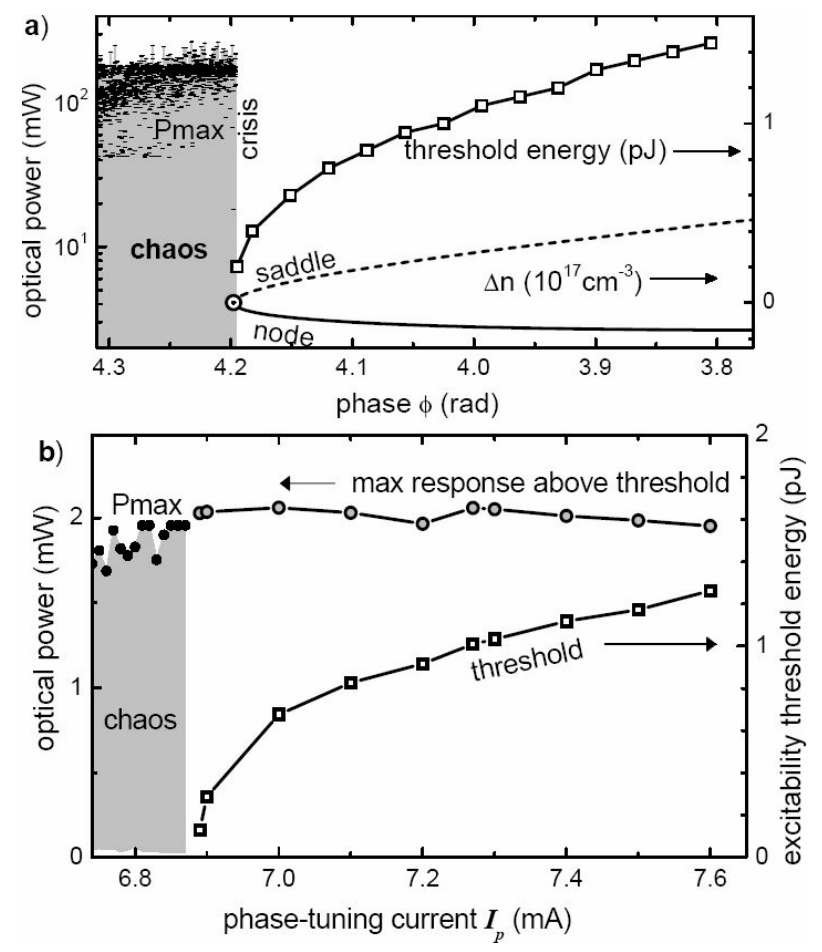

Figure 4.4: Excitability threshold versus distance from the boundary crisis. a) Calculation $\left(I_{D F B}=40 \mathrm{~mA}, I_{A}=28 \mathrm{~mA}\right)$. SN bifurcation $(\odot)$ and boundary crisis occur at feedback phase $\phi=4.1936$ and $\phi_{c}=4.1930$, respectively $\left[\mathrm{UKR}^{+} 06\right]$. Rectangles: stimulus energy at threshold, scattering dots left of $\phi_{c}$ : maximum device output in the chaotic regime computed over a time of $10 \mathrm{~ns}$, lower solid and dashed lines: carrier inversion $\Delta n$ on node and saddle, respectively, measured relative to its value at the $\mathrm{SN}$ bifurcation. b) Measurement $\left(I_{D F B}=41.7 \mathrm{~mA}, I_{A}=49.5 \mathrm{~mA}\right)$. Meaning scattering dots as in a), circles and rectangles: maximum spike strength and stimulus energy at threshold under superthreshold stimulation are inserted from Ref. [Bet03]. Note that the power output right after the device edge is plotted in the calculation, while the experimental signal is attenuated by the various optical elements of the setup before detection. 


\subsection{Excited chaotic transients}

A distinct finding, both in experiment and theory, is that the stimulation threshold strongly declines when the device is set closer to the boundary crisis, whereas the strength of the dominant response spikes stays practically constant. Closest to the SN bifurcation, an optical power as low as 100 fJ is sufficient for excitation. The calculation verifies that the threshold is determined by the saddle-node separation (Fig. 4.4a), in full agreement with the role of the stable saddle-manifold as separatrix. Various facts clearly signify that the excitable dynamics of the system close to the boundary crisis is correlated with the former CA. First, the experimental spike heights agree very well with the maximum output fluctuations of the device for $I_{P}<I_{c}$ and no injection (Fig. 4.4b). Second, in the histogram of the excited state one can observe reminiscent of distribution of chaotic motion. This distribution is convoluted with single peak distribution of stationary state, see Fig.4.2b. Third, the power spectrum of the chaotic attractor and power spectrum of the excited state are practically identical (Fig. 4.2a). Fourth, as will be detailed in the remainder, the transient spike trains subsequent to stimulation are highly irregular in frequency and amplitude.

Fig. 4.5 summarizes calculated and experimentally detected response transients close to the boundary crisis. The cw state prior to excitation is characterized by a power as well as an optical phase. The latter is random in the measurements because of a phase drift between successive excitation steps due to unavoidable experimental noise. The two transients in Fig. 4.5a are computed for the same stimulus strength but slightly different initial phases. The spike trains coincide only initially but diverge after longer times. One transient (grey) approaches equilibrium already after $3 \mathrm{~ns}$, while the other one (black) keeps spiking as long as $20 \mathrm{~ns}$. Such extreme sensitivity on the initial conditions is a clear fingerprint of chaotic dynamics associated with the motion along the CS. Indeed, also in the calculations, the excited spike sequences resemble very well the power fluctuations in the CA just before the boundary crisis. Furthermore, as can be tracked in the numerics, the irregular spiking always stops when the trajectory passes the separatrix close to the saddle-focus.

The sampling technique used experimentally provides one data point per stimulation event in the power-time diagram. Repeating the time scans sufficiently often yields then the probability that the device emits a certain power at given time. An example of such measurement is displayed in Fig. 4.5b. Chaotic transients differ from each other after a time of the order of the largest inverse Lyapunov exponent. Consistently, a couple of quasi-deterministic spikes is seen at very early times in Fig. 5b. Later spikes 

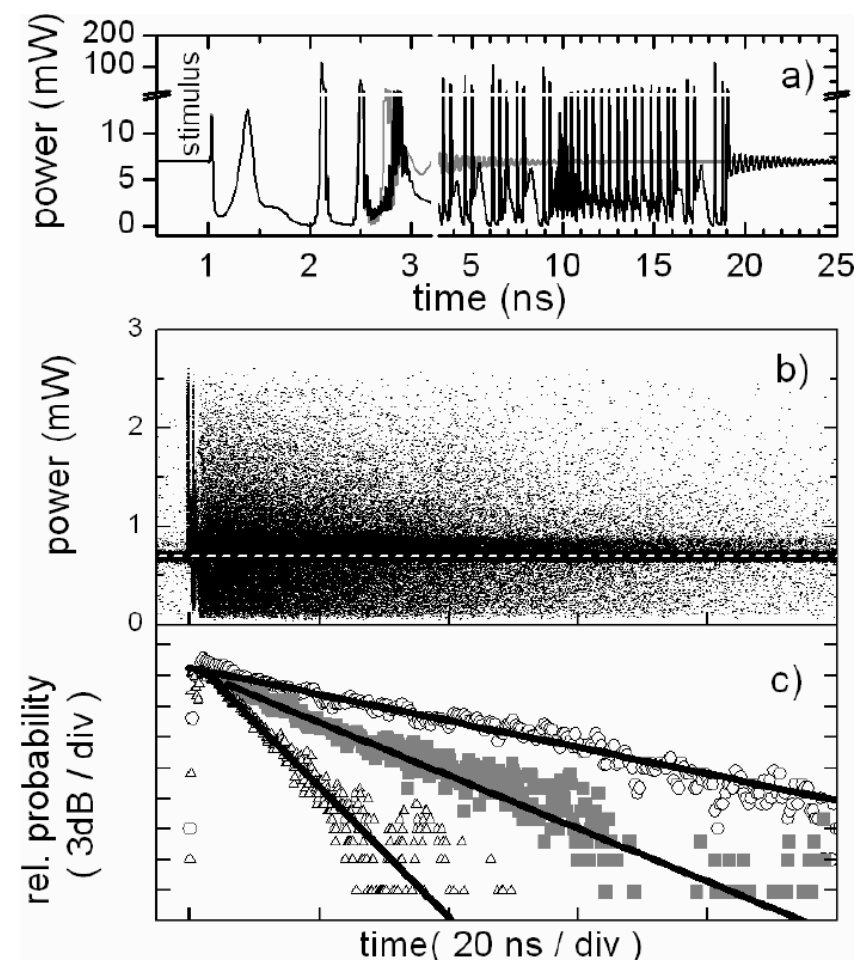

Figure 4.5: Transients after superthreshold excitation. a) Comparison of two calculated responses (black and grey, $\phi=4.15$ ). The stimuli differ only by the initial phase difference between internal and injected fields. Initially, the two transients are indistinguishable. At later times, however, they diverge resembling the extreme sensitivity of chaos on initial conditions. One transient (grey) approaches equilibrium after $3 \mathrm{~ns}$ ). The other one stays nearly 20 ns on the chaotic ruin. b) Superposition of 500 measurements with the sampling oscilloscope (stimulus repetition rate $12.5 \mathrm{MHz}$ ). The point of operation (phase current $6.9 \mathrm{~mA}$ ) is close to the crisis. and not all transients do return to the rest state within the $80 \mathrm{~ns}$ repetition period. c) Probability of presence in the CS versus time after stimulation for three different distances from the boundary crisis $I_{P}-I_{c}$ : $0.04 \mathrm{~mA}$ (triangles), $0.02 \mathrm{~mA}$ (squares), and $0.01 \mathrm{~mA}$ (circles). Lines: singe-exponential fits.

are irregularly spaced and yield thus only a cloud of uncorrelated dots. The latter starts few nanoseconds after stimulation, which is in fair agreement with the numerically calculated Lyapunov exponents [UKR $\left.{ }^{+} 06\right]$. The power distribution along a vertical line in Fig. $4.5 \mathrm{~b}$ is a superposition of the narrow Gaussian related to the rest state and a much broader band due to the chaotic response. In detail it is shown in Fig.4.2b. The area below the 


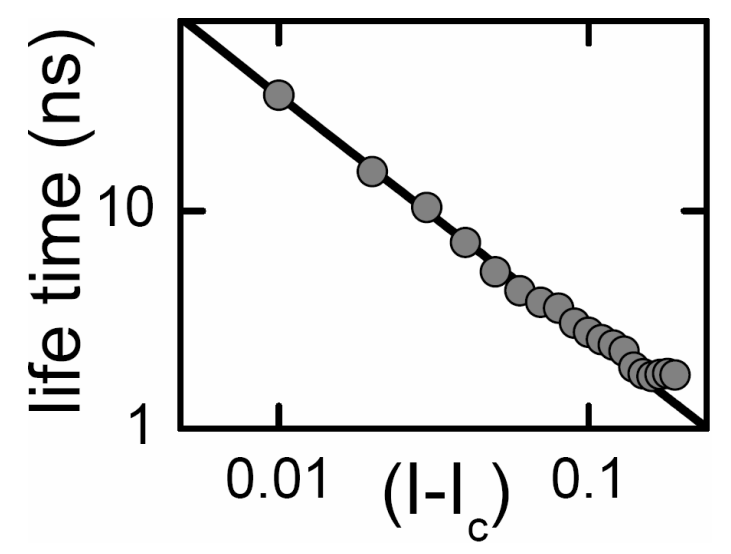

Figure 4.6: Mean lifetime $\tau$ on the CS versus $I_{P}-I_{c}$. Solid line: inversepower function with exponent $\gamma=1$.

wide band is a measure for the probability to find the system still in the $\mathrm{CS}$ at this time. The plots in Fig. 4.5c reveal that this probability decays strictly single-exponential with a time constant $\tau$ representing the mean lifetime in the CS. This lifetime becomes shorter with increasing distance from the boundary crisis. As long as the distance is not too large, $\tau$ obeys an inverse-power law $\sim\left(I_{P}-I_{c}\right)^{-\gamma}$ with a critical exponent $\gamma \approx 1$ (Fig. 4.6). Beyond $I_{P}-I_{c} \approx 0.1 \mathrm{~mA}$, saturation sets on and $\tau$ approaches a constant value of about 1.8 ns. In parallel, as already noted above, the response of the device becomes increasingly dominated by a single spike and is thus fully analogous to the previously observed excitability at a homoclinic bifurcation [WBRH02].

A time-independent escape rate $1 / \tau$ from the $\mathrm{CS}$ is a generic property of chaotic transients [GOY86; GORY87; $\mathrm{DRC}^{+} 89$; $\mathrm{SDG}^{+}$91; ASY06]. Inversepower laws are well established for one- or two-dimensional maps [GOY86; GORY87] and have been experimentally verified in a nonautonomous mechanical system $\left[\mathrm{DRC}^{+} 89 ; \mathrm{SDG}^{+} 91\right]$. Three dimensions have been treated theoretically only very recently [ASY06]. The present study provides direct evidence that an inverse-power law remains valid also for the boundary crisis of a high-dimensional CA in a continuous and autonomous system. The critical exponent of one-dimensional maps is generically 0.5 [GOY86; GORY87], a three-dimensional example has yielded 1.5 [ASY06], and those of twodimensional maps lie in between. It has been argued [GOY86; GORY87] that the critical exponents should increase with the dimension of the chaos. Our results do not confirm this conjecture: In the continuous system under study, $\gamma$ is close to 1 although the estimated dimension of the CA is as large as 3.5 . 


\subsection{Influence of noise}

Noise in the presence of a CS can also induce chaotic behavior [LLBS03]. In order to investigate the role of noise in the multi-section laser, we have repeated the measurements without external excitation. Indeed, close to the boundary crisis, random jumps into chaotic transients are observed, wide wings in Fig.4.7. However, in marked contrast to the excitable dynamics, their probability decreases very rapidly with the distance from the crisis and becomes as small as $10^{-4}$ already at an excess current of $0.01 \mathrm{~mA}$. Noise-

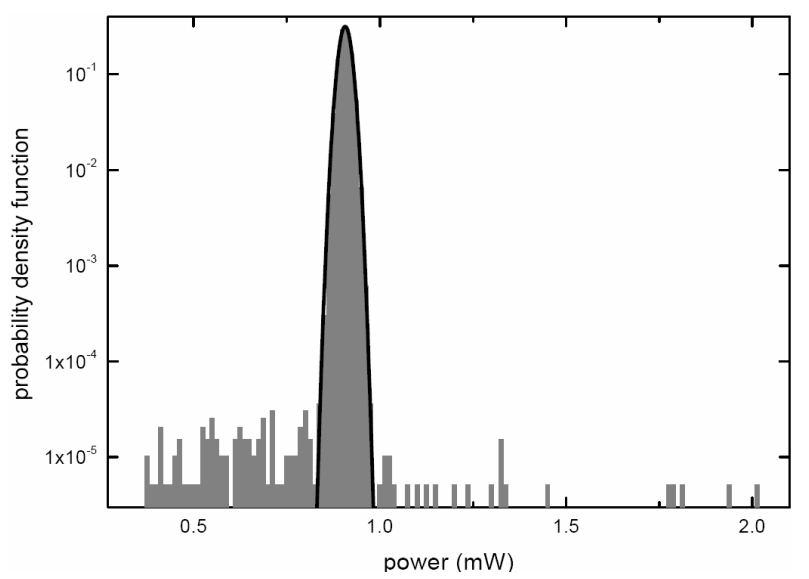

Figure 4.7: Histogram of stationary state close to the boundary crisis $I-$ $I_{C} \approx 0.01 \mathrm{~mA}$, Gaussian approximation (solid line). Side wings indicate noise induced wandering on the chaotic saddles. Ratio of area under the wings and the main peak is $0.6 \times 10^{-4}$.

induced dynamics is thus of minor importance in our case. In particular, the few data points in Fig. 4.5b before application of the excitation pulse are not a result of noise but correspond to chaotic transients which last longer than the 200 ns separation between subsequent stimuli. Such an extremely extended response is a characteristic feature of a CS: It involves trajectories wandering arbitrarily long in the saddle before approaching an attractor.

\subsection{Conclusion}

We have presented a detailed experimental and numerical investigation of the dynamics close to a boundary crisis. It is shown experimentally that the chaotic motion is destroyed in a boundary crisis. The escape from the underlying chaotic saddles is strictly single-exponential and validity of an 
inverse-power law for the escape time is found. Despite of high dimensionality, the critical exponent is close to unity. The excitation of the chaotic transient exhibits a distinct threshold as well as a refractory time and, sufficiently far from the boundary crisis, the standard response of excitable systems is recovered. The relaxation times of the carrier-photon dynamics in the laser are in the sub-ns range. A striking finding from a practical point of view is therefore that the device is capable of emitting pulses with a delay on a two orders of magnitude longer time scale. 


\section{Chapter 5}

\section{Coherence resonance at a Hopf bifurcation}

This chapter addresses the influence of noise on the dynamics close to the bifurcations investigated in the previous chapter. Noise induced regularity phenomena such as a coherence resonance are studied at the onset of a Hopf bifurcation. A generic model supplements the experimental findings.

\subsection{Noise induced regularity}

The paradigmatic and pioneering example of noise induced motion is that of stochastic resonance (SR) in bistable systems [BSV81], which had been originally proposed as a mechanism to describe the observed periodicity of the Earth's ice ages. Here, a dynamical system with two stable fixed points is subject to the action of a periodic forcing and noise. The periodic forcing is sufficiently weak that, by itself, it can not overcome the separation barrier between the stable fixed points. The system oscillates around one of them, determined by the initial conditions. The presence of noise, however, allows the system to make random excursions and visit both fixed points. The jumps between the fixed points occur when the impact of the external forcing sufficiently lower, the separation barrier. The signal-to-noise ratio (SNR), appropriate measures of the signal coherence, pass through a maximum at an optimal noise strength when the noise-controlled time scale of the system matches the period of the external signal. A related phenomenon is that of coherence resonance (CR) by which a nearly periodic signal is generated under the effect of the fluctuations in systems which lack an external signal. Again, the periodicity is optimal (resonance) for a given value of the noise intensity [PK97; LNK98; GDNH93]. 
Coherence resonance can manifest itself in quite different systems due to different mechanisms. In excitable systems, the essential role of slow and fast dynamics, and the nonuniformity of oscillations was emphasized in [LGONSG04]. This approach was successful in interpreting CR in neuron models [PK97; LNK98; LSG00]. CR in excitable systems was experimentally confirmed in a laser diode with optical feedback [GGBT00] and in electronic circuits [PHYS99]. CR has also been observed in bistable systems such as in simulations of the FitzHugh-Nagumo model [LSG00], and both in simulations and experiments with the chaotic Chua model [CMT01; PTMC01]. Wiesenfeld [Wie85] has demonstrated that power spectrum of a system observed after a bifurcation point can, nevertheless, be visible even before the bifurcation actually occurs if noise is present. We thus observe a noisy precursor of the bifurcation. Let us suppose that noise induces a peak of height $H$ at the frequency $\omega_{p}$ in the power spectrum, so that the noisy precursor of a bifurcation is observed.

In order to measure the coherence of the system at the noise induced peak one can define the signal-to-noise ratio as $\beta=H / W$ [GDNH93], with the peak's relative width $W=\Delta \omega / \omega_{p}$, which is the inverse of the familiar quality factor $Q$. Coherence resonance at a noisy precursor manifests itself when SNR displays a maximum as a function of the noise level. Coherence resonance at noisy precursors can be explained [NSS97] by the interplay of the constructive effect of noise as promoting coherent precursors and by the well-known destructive effect as eliminating order.

Noisy precursors of period doubling and torus bifurcations have been numerically investigated in Ref.[NSS97]. Resonance-like behavior close to the onset of a supercritical Hopf bifurcation has been found in computer simulations [HX99; KL00; RTAW01; JC02] and observed experimentally for plasma waves [DWK99; LJ95] and electro-chemical reactions [KHSP03]. However, in all these studies, the question how the type of the bifurcation controls the noise dynamics and, in particular, whether this manifests in the existence of CR has not been explicitly addressed.

In this work, we examine the case of a $\mathrm{CR}$ at a Hopf bifurcation. We demonstrate that noise induced resonances occur for both the subcritical and supercritical type. However, the specific response is crucially determined by the character of the bifurcation. 


\subsection{Experimental characterization of bifurca- tions and states}

This section is devoted to characterization of the bifurcations close to which noise induced dynamics is studied.

\subsubsection{Experimental setup}

In order to characterize noise induced oscillations, the laser with active optical feedback is connected to an electrical spectrum analyzer (R\&S FSP 9/40GHz) with bandwidth of $40 \mathrm{GHz}$ by a u2t photo diode. Electrical broadband noise

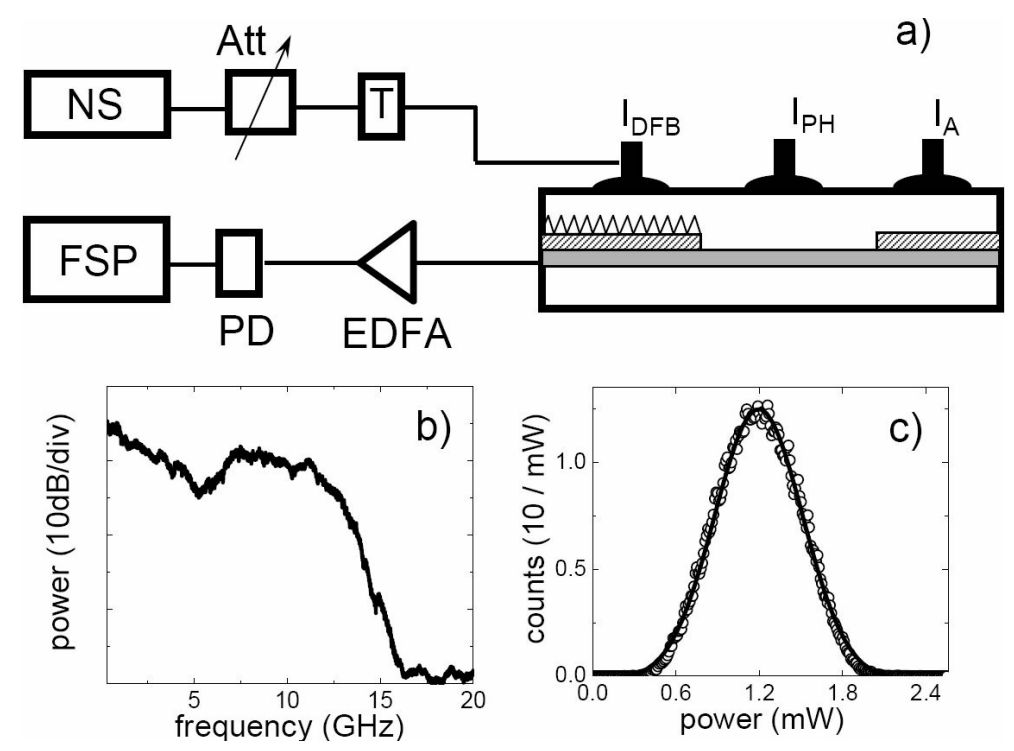

Figure 5.1: a) schematics of the multi-section laser device and the experimental setup. NS - an electrical noise source, Att. - a tunable attenuator, $\mathrm{T}$ - a bias tee, PD - a photo diode, ESA - a power spectrum analyzer, EDFA - an erbium doped fiber amplifier. b) a power spectrum of electrical broadband noise; c) an intensity distribution of noise (open dots), Gaussian approximation (solid line).

is generated by a $50 \Omega$ resistor at room temperature and then amplified by three electrical amplifiers (SHF100CP) with $25 \mathrm{GHz}$ bandwidth. The power spectrum of the noise (Fig.5.1 a)) is practically constant in the relevant frequency range. The noise is added to the laser injection current $I_{D F B}$ and thus imprinted in the carrier density. In order to avoid a shift of the operation point, the dc component is filtered by a broadband bias tee (SHFBT45) with 
a bandwidth of $45 \mathrm{GHz}$. While the average of the noise signal itself is zero, the noise intensity is Gaussian distributed (Fig.5.1 b)) with a mean value adjusted experimentally by a tunable attenuator (HP8494B/11dB) with an accuracy of $1 \mathrm{~dB}$. The maximally accessible value of electrical noise is $D=$ $-20 \mathrm{dBm} / \mathrm{GHz}$.

\subsubsection{Noisy precursors}

As shown in Chapter 2 the laser with optical feedback is capable of two stable self-pulsations with distinctly different frequencies determined by their specific physical origin. The beating of two longitudinal compound-cavity modes (MB) gives rise to pulsations with frequencies $f>20 \mathrm{GHz}$. Here, we focus on the low frequency pulsations $f \leq 12 \mathrm{GHz}$ arising from undamped relaxation oscillations (URO) due to dispersive $\mathrm{Q}$ switching. URO can be born in both types of Hopf bifurcations $\left[\mathrm{BBK}^{+} 04\right.$; Bau05], allowing us to investigate the influence of noise under otherwise identical physical conditions (Fig.5.2(a1),(b1)). The relaxation-oscillation self-pulsations are also born in a supercritical torus bifurcation when the higher-frequency mode-beating pulsations are already present (Fig.5.2(c1)). In order to elucidate the influence of noise, the injection currents are adjusted close to the respective bifurcation point, shown by arrows in Fig.5.2. Even in the absence of external noise, as documented by the weak features in the power spectra of Fig. 5.2, precursors of the pulsations are observed for all three bifurcations. Those precursors are induced by the intrinsic noise of the device. When adding external noise, the precursors develop into pronounced resonance features (Fig.5.2 (a2),(b2) and (c2)).

\subsection{Coherence resonance close to a Hopf bi- furcation}

Eventually, the noise creates merely fluctuations in the laser emission, as indicated by an increased noise floor in the power spectrum. To analyze the spectral response quantitatively, the data are fitted by a Lorentzian lineshape function with peak frequency $\omega_{p}$, a full width at half maximum $\Delta \omega$ and peak height $H$.

The width $\Delta \omega$ translates by Fourier transformation into a correlation time $\tau_{c} \sim 1 / \Delta \omega$. There is only a very small shift of the peak frequency so that the quality factor $Q=\omega_{p} / \Delta \omega$ is an inverse function of the width. As mentioned above, a measure characterizing the noise-induced response is the 

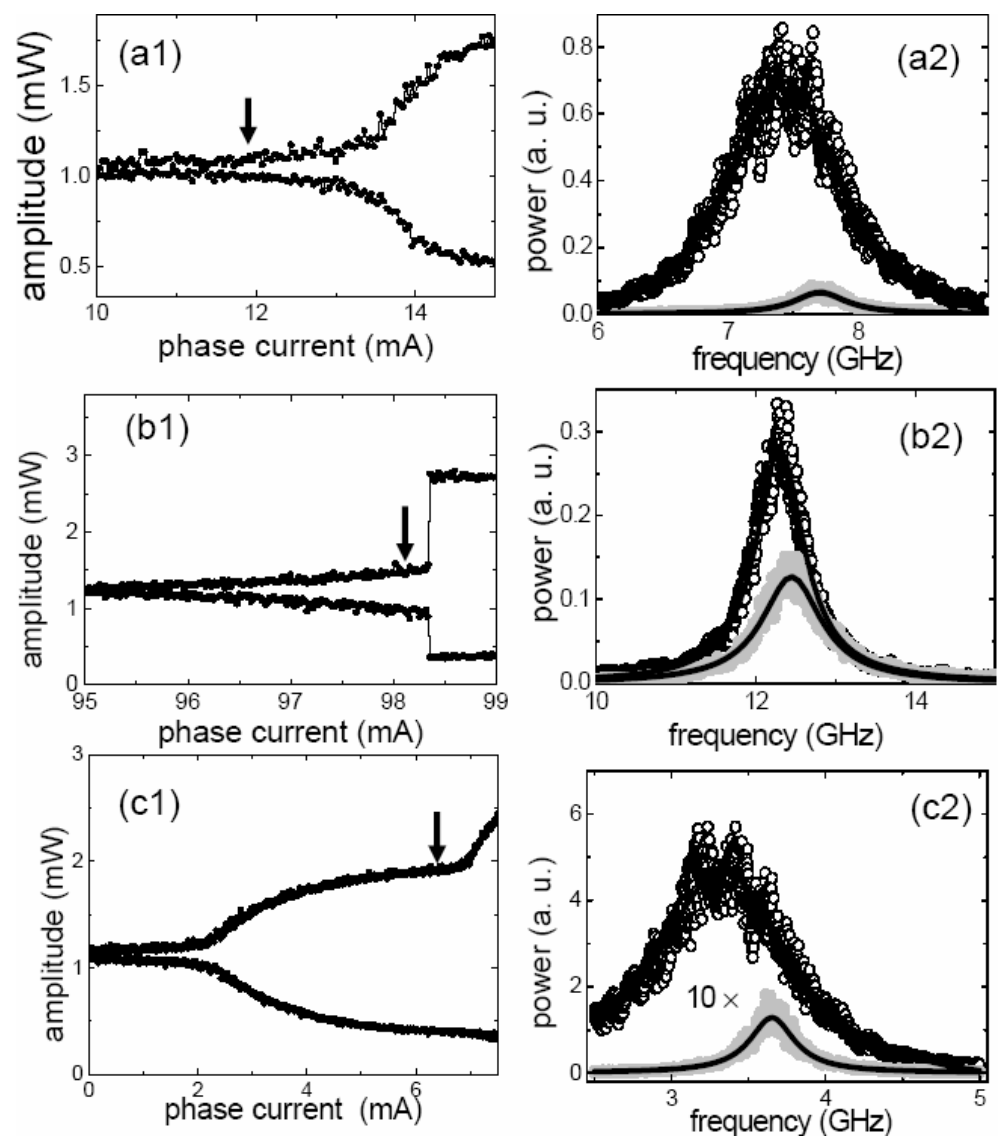

Figure 5.2: Left column: maximum $\left(P_{\max }\right)$ and minimum $\left(P_{\min }\right)$ output power versus phase current. Right column: power spectra of the device under the influence of noise. (a2) Before the supercritical Hopf bifurcation without external noise (lower plot) and at optimum noise level (upper plot), solid lines are fits with a Lorentzian shape $\left(I_{D F B}=70 \mathrm{~mA}, I_{A}=5.62 \mathrm{~mA}, I_{P H}=11.8 \mathrm{~mA}\right)$. The same for the subcritical case (b2) $\left(I_{D F B}=70 \mathrm{~mA}, I_{A}=30.6 \mathrm{~mA}, I_{P H}=98.2\right.$ $\mathrm{mA})$ and the torus bifurcations (c2) $\left(I_{D F B}=41.7 \mathrm{~mA}, I_{A}=47 \mathrm{~mA}, I_{P H}=6.1\right.$ $\mathrm{mA})$. Arrows correspond to operating points.

signal-to-noise ratio[GDNH93; NSS97; LGONSG04]

$$
\beta=H Q=H \omega_{p} / \Delta \omega
$$

As seen in the Fig. 5.3, $\beta$ is a non-monotonic function of the noise intensity, demonstrating the existence of an optimum noise level. However, qualitative differences between the bifurcations turn out, when height and width are considered. In the supercritical case, $H$ and $\Delta \omega$ increase monotonically, although with different slopes. In particular, the height saturates at higher 
noise intensities, as the system nonlinearities limit the oscillation amplitude (Fig. (a), (b)). In contrast, the width exhibits a clear minimum at a certain

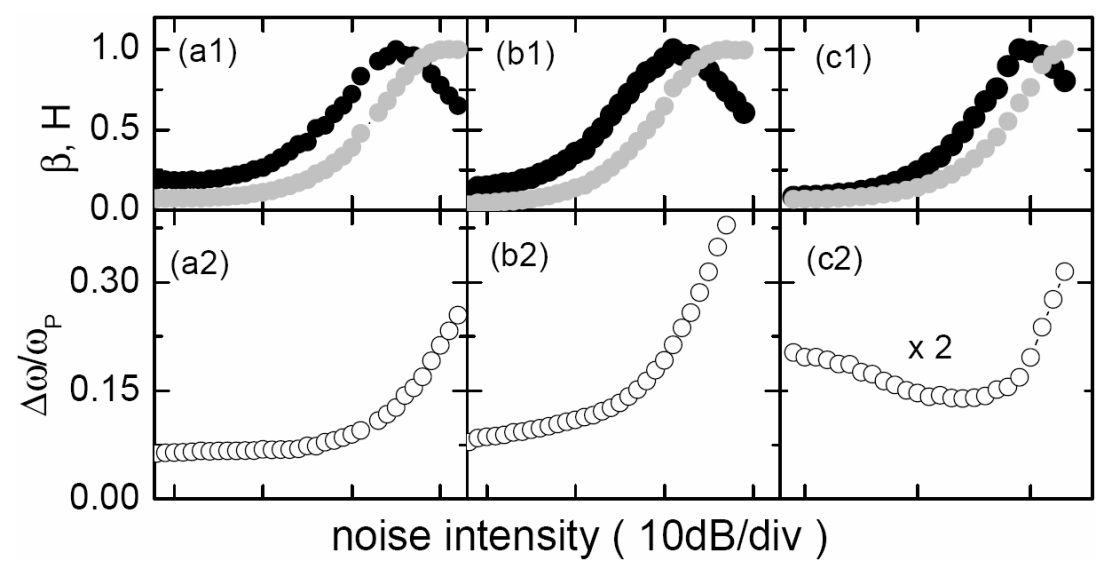

Figure 5.3: Peak height (gray dots), normalized width (open dots), and signal-to-noise ratio (black dots) versus noise intensity as deduced from the power spectra by Lorentzian fits. Note the log scale. (a1,2) Supercritical bifurcation, (b1,2) Torus bifurcation, and (c1,2) Subcritical bifurcation. Maxima of curves are normalized to unity.

noise intensity for the subcritical bifurcation (Fig. 5.3(c)). In the time domain, this means a maximum of $\tau_{c}$, similarly to the CR of excitable systems [PK97]. However, there is a marked difference: The noise-induced peak is already present at infinitely small noise intensities in the present case, whereas the threshold of excitable systems produces an abrupt occurrence at a certain noise level.

Locating the system on the stable limit cycle close to a supercritical torus bifurcation and adding noise, the result is qualitatively the same as for the Hopf bifurcation, (compare Figs. 5.3 (a1) and (b1)) for the case of a stable focus. This suggests that, irrespective of the specific oscillator involved, the type of the noise resonance is determined by the subcritical or supercritical character of the bifurcation. The succeeding analysis demonstrates that the above observations are indeed of much wider reach, as they uncover generic scenarios close to a Hopf bifurcation. 


\subsection{Generic model}

Onset and enhancement of self-sustained oscillations can be successfully modeled by the complex differential equation

$$
\dot{z}=-i \omega_{0} z-z F(z),
$$

used in many different contexts. Here $z=x+i y$ is a complex amplitude, $\omega_{0}$ is the eigenfrequency. In fact, the model (5.2) is a normal form for the Hopf bifurcation; examples of applications range from the Van der Pol equation to the Lang-Kobayashi mean field equations [SPM04]. The bifurcation scenario depends on the non-linear function $F(r)$. For the supercritical Hopf bifurcation, $F(r)$ takes the form

$$
F_{1}(r)=-a_{1}+r^{2}+r^{4}
$$

with $r=|z|=\sqrt{x^{2}+y^{2}}$; at $a_{1}=0$ the equilibrium at $|z|=0$ gets destabilized, and the stable limit cycle is born from it. Alternatively, the subcritical Hopf bifurcation is rendered by

$$
F_{2}(r)=-a_{2}-r^{2}+r^{4}
$$

Here, the increase of the parameter $a_{2}$ results, first, in the birth of the finite amplitude oscillatory state at $a_{2}=-1 / 4$ and, second, in the destabilization of equilibrium at $a_{2}=0$. The former event is the saddle-node (tangent) bifurcation which creates on the phase plane two limit cycles: the stable and the unstable one. The latter event is the Hopf bifurcation: the unstable limit cycle shrinks to the equilibrium which, thereafter, inherits its instability. In the parameter range between two bifurcations the system has two attractors: the equilibrium and the stable limit cycle.

We set $\omega_{0}=1$ and fix the values $a_{1}=-0.05$ and $a_{2}=-0.257$, respectively. This choice adjusts the systems before the birth of self-sustained oscillations, $z=0$ being a stable fixed point and $d r / d t<0$ everywhere outside this point. In the first operation point a stable focus "almost" gives birth to a limit cycle. All trajectories are spirals approaching to the fixed point (Fig.5.4(a)). In the second case the system is close to a saddle-node bifurcation of periodic states, after which a stable fixed point and a stable limit cycle coexist. If the system trajectories start far enough from the fix point one observes monotonic spiraling to the origin. However, there is a region where spiraling is slowing down and the trajectories stay a long time in the region of impending limit cycle (Fig.5.4(b)). Below, we will argue why the presence of this region is responsible for the minimum in the spectral width. 

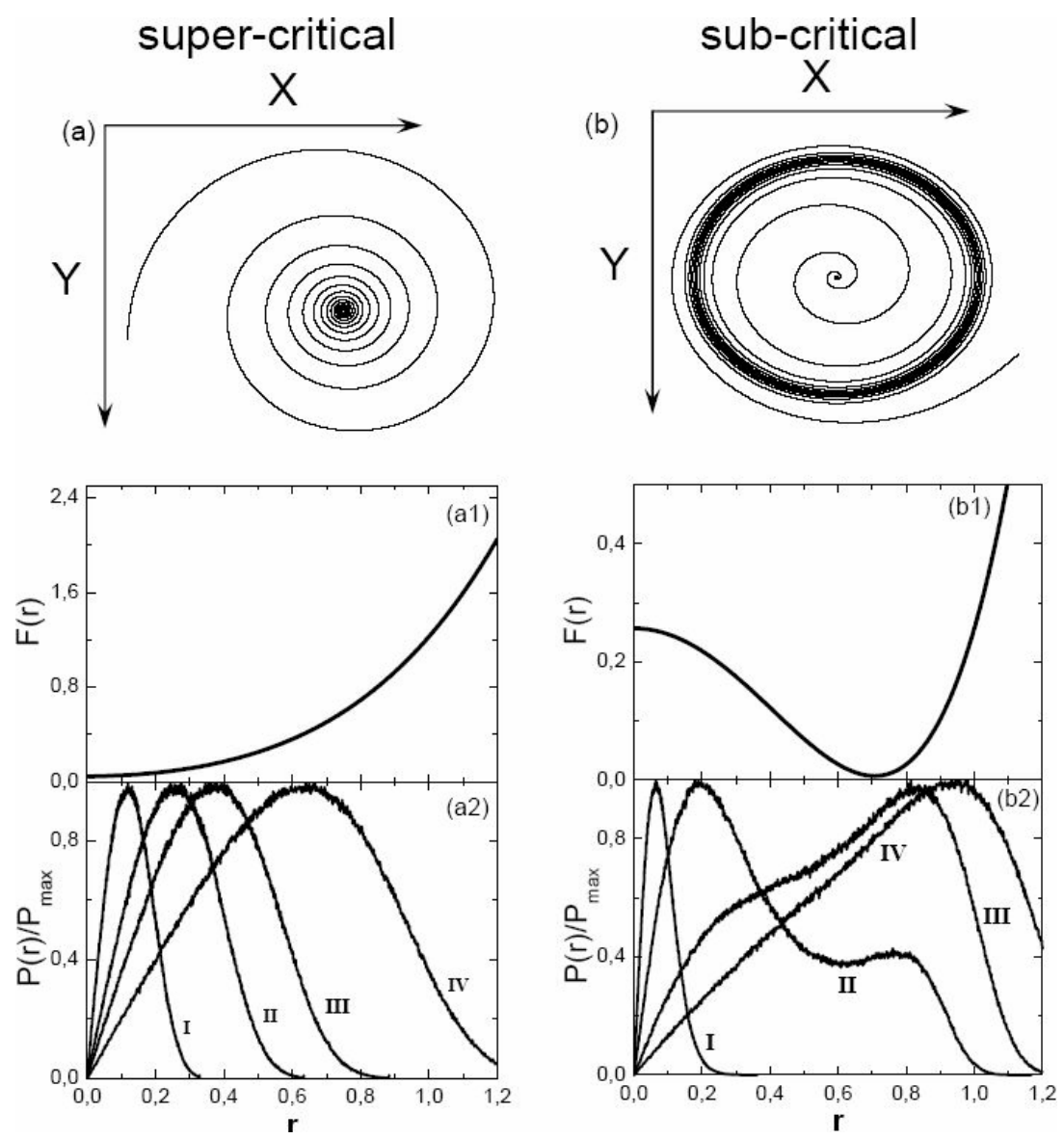

Figure 5.4: (a), (b) calculated trajectories. (a1), (b1) local damping F(r). Normalized distribution $P(r)$ for different level of noise. (a2) I - $\mathrm{D}=0.0001$, II - $\mathrm{D}=0.0016$, III - $\mathrm{D}=0.008$, IV - $\mathrm{D}=0.13$. (b2) I - $\mathrm{D}=0.0001$, II - $\mathrm{D}=0.0016$, III - D $=0.008, \mathrm{IV}-\mathrm{D}=0.0625$; Left column: supercritical case; Right column: subcritical case.

We rewrite the model (5.2) in polar coordinates:

$$
\begin{aligned}
\dot{r} & =r F(r), \\
\dot{\varphi} & =\omega_{0} .
\end{aligned}
$$

Here $r=\sqrt{x^{2}+y^{2}}, \varphi$ is a phase, and $\omega_{0}$ is the eigenfrequency. If $F(r)$ would be a constant what is valid for linear oscillator than equations $(5.5,5.6)$ can be solved analytically and linewith is entirely determined by $F, \Delta \omega=\pi F$. So for linear oscillator $F(r)$ is related to the spectral linewidth. We consider only amplitude equation (5.5) because noise in the phase equation plays no 
a significant role:

$$
\dot{r}=r F(r)+\sqrt{2 D} \xi_{r}(t)
$$

Here $\xi_{r}(t)$ represents a non-white multiplicative noise with intensity $D$.

The difference between super- and subcritical cases can be explained by the following arguments. First, the damping of (5.7) is determined by function $F(r)$. Second, since the amplitude $r$ is distributed due to noise, its mean value $\langle r\rangle$, as a function of $D$, moves from small to large values when the noise becomes stronger. In other words, for different intensities of noise, oscillations occur in regions with different local damping $F(r)$.

For super-critical case $F(r)$ is monotonically increasing function of $r$ (Fig.5.4(a1)) and, consequently, of the noise intensity $D$. In the region of weak noise, it almost does not grow, and we observe noise induced oscillations with small amplitude and nearly the same coherence properties. This can be related to phase dynamics. After $F(r)$ is noticeably increased, the coherence gets lost.

For sub-critical case the damping $F(r)$ is a non-monotonic function of $r$ (Fig.5.4(b1)): in the range of moderate amplitude $r$ it is decreasing. Accordingly, when the trajectory of the system visits the region with low damping, we observe time intervals of oscillations with high coherence. For large noise intensity, $F(r)$ is significantly increased, and coherent oscillations do not occur.

We assume that the spectral width is governed by the effective damping $<F(r)>$ at fixed noise intensity $D$, which is defined as expectation value of $F(r)$ :

$$
<F(r)>=\int F(r) P(r) d r
$$

Here $P(r)$ is the stationary distribution of the amplitude fluctuation. One can expect monotonic behavior of the effective damping versus noise intensity for supercritical Hopf bifurcation, whereas for subcritical case minimum should be observed.

In order to verify this statement the model (5.7) was solved numerically by a stochastic Heun method [KP06] with time step $10^{-3}$. Distribution of the amplitude $P(r)$ for different noise intensities are shown in Fig.5.4 (a2), (b2). One observes shift of the distribution maximum to higher amplitude with increasing of noise intensity. Bimodal distribution in the subcritical case is due to nonmonotonic dependence of the damping on coordinate. Spectral width was obtained from the computed power spectra fitted by Lorentzian functions. In calculation of the effective damping $\langle F(r)>$ the interval 
of integration is chosen so that $\mathrm{P}(\mathrm{r})$ is larger than $10^{-2}$. Fig.5.5 exhibits the effective damping $\langle F(r)\rangle$ and the spectral width $\Delta \omega$ versus noise intensity. In case of supercritical Hopf bifurcation monotonic growth of both the width and the effective damping is observed. Minimum in the effective damping as well as in the spectral width is observed for subcritical case (Fig.5.5(b)). Quantitative disagreement is visible and can be explained by that fact that the spectral width is not exactly defined by effective damping. Strict analytical treatment of the model is performed in Ref. [KLGZ06].
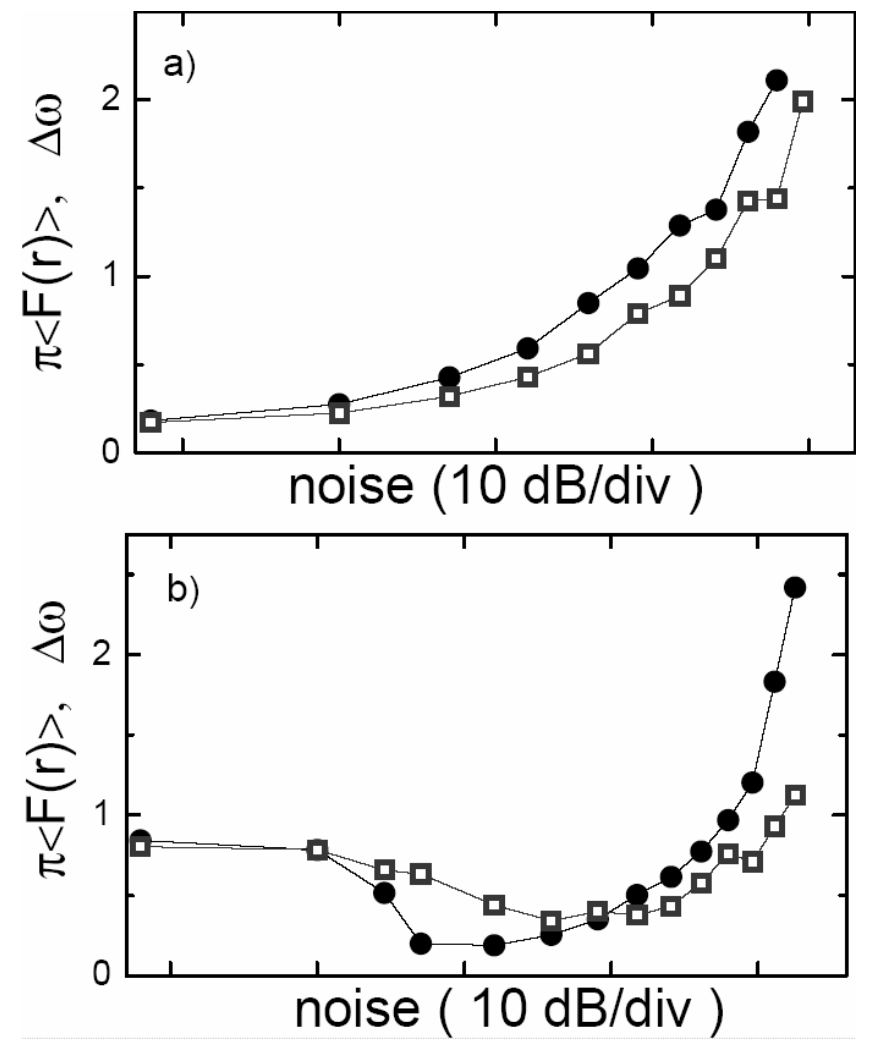

Figure 5.5: Results of numerical simulation: spectral width $\Delta \omega$ (dots) and mean local damping $\langle F(r)\rangle$ (squares); a) supercritical Hopf bifurcation, b) subcritical Hopf bifurcation.

One can summarize that the nonlinearity in the amplitude equation is related to nonlinearity in damping. Before the supercritical Hopf bifurcation the damping is minimal in the fixed point, whereas before subcritical bifurcation the minimal damping values take place outside the fixed point, in the region where impending limit cycle arises. Consequently, the main ingredient for the difference between the manifestation of coherence resonance is the difference in the form of dependence of damping on the coordinate. 


\subsection{Conclusion}

We have demonstrated that resonance phenomena driven by noise are a general concomitant to a Hopf bifurcation. However, while the existence of an optimum noise level is a common feature for both types of bifurcations, the physics behind the resonance effect is qualitatively different. In the supercritical case, the increase of the signal-to-noise ratio is produced by the spectral peak height, that is, by an increase of the oscillation amplitude. The width is initially only slightly affected, but increases steeply for stronger noise, weakening the coherence. Resonance-like behavior originates from the competition between the growth of height and width. In contrast, for the subcritical type, the spectral width itself exhibits a minimum. Here, the noise improves, indeed, the quality factor as well as the temporal coherence of the oscillation transients. In a strict sense, only this kind of response represents truly "coherence resonance". 


\section{Chapter 6}

\section{Conclusions}

This thesis reports on the experimental investigation of the self-organization in a single-mode semiconductor laser with ultrashort optical feedback.

The dynamical properties of the laser with ultrashort optical feedback are governed by two main parameters: a strength and an optical phase shift of the feedback. First, laser with passive feedback (PFL) is investigated. Varying of feedback phase gives rise to self-pulsation. Two types of self-pulsation are seen undamped relaxation oscillations (URO) and beating of a pair of longitudinal modes (MB pulsation). It has been experimentally shown that both pulsations appear in a Hopf bifurcation. Transition to higher order instabilities, period doubling bifurcation, is observed as well. However, the period doubling sequence does not evolve into a chaotic attractor probably due to collision of the attractor with a saddle. Chaotic motion can be obtained with increasing of the feedback strength. The laser with active optical feedback (AFL) possesses an ability to tune the feedback strength and the feedback phase separately. At higher feedback strength the quasiperiodic route to chaos is found. An internal crisis is responsible for the expansion of the chaotic attractor in the phase space. Chaotic motion is destroyed by a boundary crisis when the chaotic attractor collides with a saddle. Destabilized chaotic attractor, chaotic saddle (CS), appears behind the crisis. Chaotic transients (CT), a typical fingerprint of CS are observed experimentally by exciting the system with an external short optical pulse. The lifetime of the CT scales with the distance to the crisis as power law with critical exponent. Despite high dimensionality this exponent is equal to unity. The excitation of the chaotic transient exhibits a distinct threshold as well as a refractory time and, sufficiently far from the boundary crisis, the standard response of excitable systems is recovered.

In Chapter 5 we have investigated the influence of external Gaussian noise close to the onset of the Hopf bifurcations. Noise-induced oscillations appear 
as a Lorentzian-shaped peak in the power spectrum. The coherence factor defined by the product of height and quality factor exhibits non-monotonous behavior with a distinct maximum at a certain noise intensity for both types of Hopf bifurcations. Thus, both types of Hopf bifurcations exhibit coherence resonance. Besides these similarities, the measurements reveal also qualitative differences between the two cases. Whereas the width of the noise induced peak increases monotonically with noise intensity for the supercritical bifurcation, it traverses a pronounced minimum in the subcritical case. These experimental findings are examined in terms of general models for the noise driven motion close to bifurcations. 


\section{Appendix A}

\section{Probability distribution function}

It is natural to seek probabilistic descriptor when deterministic systems are in a state of dynamical chaos. Such statistical tools are widely used in the random excitation of linear systems. However, probabilistic mathematics for chaotic dynamics of nonlinear systems are not readily available. One exception is the case of systems governed by a first order-difference equation or map.

For a first-order map, the probabilistic description involves a function $P(x)$ called the probability density function $(P D F)$, where $x$ is the state variable that governs the map

$$
x_{n+1}=F\left(x_{n}\right) \text {. }
$$

Because, $x$ is a continuous variable, $P(x) d x$ is the probability that the dynamical orbit will occur in $(x, x+d x)$. The domain of the variable $x$ over which $P(x) \neq 0$ is sometimes called the support of the probability measure. One complication in chaotic systems is that the support is sometimes fractal. However, in practical systems there is always a small amount of noise which tends to smooth out the fractal nature of $P(x)$.

A fairly obvious way to calculate an approximation to $P(x)$ is to divide up or partition the domain of $x$ into $N$ cells of size $\Delta x$, and then run map for several thousand iterations, counting the number of times $N_{i}$ an orbit enters the $i$ th cell. The set of numbers, sometimes called a histogram $P_{i} ; i=1, \ldots, N$, is given by

$$
P_{i}=N_{i} / N
$$

The histogram is then considered to be an approximation to $P(x)$. 
Increasingly, measurement of the probability distribution function is being used as a diagnostic tool in chaotic vibration. In general, a dynamic attractor in three-dimensional phase space would have a probability measure with three variables $P(x, y, z)$, one for the each of the state variables.

Also, modern signal processing systems often have a function (called a histogram) that will partition an interval $x_{1} \leq x \leq x_{2}$ into $N$ bins and that will count the number of times the digitalized signal lies in each bin in a given finite length data record. With suitable normalization, this procedure will yield an approximation to $P(x)$.

To function as a good diagnostic tool, a signal processing algorithm must provide qualitatively different patterns for periodic and chaotic signals. A periodic motion usually has an elliptic shape in the phase plane $(x, \dot{x})$. If the points on the orbit are subdivided and projected onto the $x$ - axis, then probability density function $P(x)$ is continuous over finite interval with singularities at the edges; that is,

$$
P(x)=\frac{1}{\pi} \frac{1}{\sqrt{A^{2}-x^{2}}}
$$

for a harmonic orbit center at the origin. ( $A$ is the maximum amplitude of the limit cycle.) For chaotic signals, the singularities often disappear and $P(x)$ looks more Gaussian and often non-Gaussian. 


\section{Appendix B}

\section{Travelling-Wave-Model}

The slowly varying envelopes $E^{ \pm}(t, z)$ of the forward and backward travelling waves obey the equations

$$
\frac{\partial}{\partial t} E^{ \pm}=v_{g}\left(\mp \frac{\partial}{\partial z}-i \beta+\frac{\alpha}{2}\right) E^{ \pm}-i v_{g} \kappa E^{\mp},
$$

where $v_{g}$ is the group velocity, $\alpha$ the absorption coefficient for parasitic losses, $\kappa$ the coupling coefficient of the DFB gratings. Boundary conditions read $E^{+}(0, t)=0$ at the antireflection coated DFB facet and $E^{-}(L, t)=$ $r_{L} E^{+}(L, t)$ at the cleaved facet of the feedback cavity. The waveguide propagation parameter $\beta$ is a constant in the passive phase tuning section. For a length $l_{P}$, it is given by

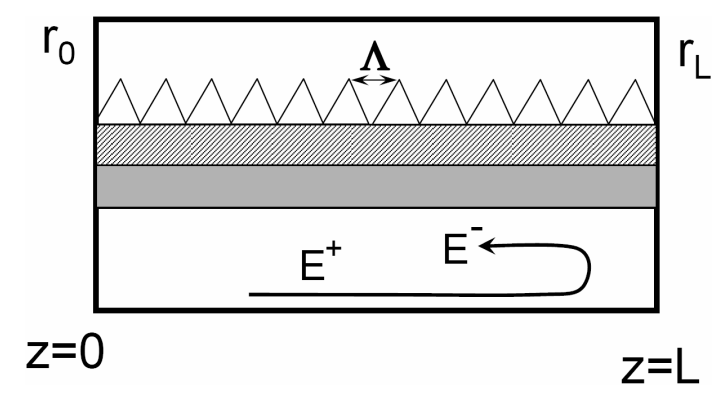

Figure B.1: Schematic of a DFB laser. $E^{ \pm}(t, z)$ - the forward and backward travelling waves, $r_{0}, r_{L}$ - facet reflectivities.

$$
\beta=-\frac{\phi_{P}}{2 l_{p}},
$$

where the phase shift $\phi_{P}$ represents one of the externally controllable bifurcation parameters of the AFL dynamics. 
In the active sections, $\beta$ is a function of $t$ and $z$ and contains the following contributions:

$$
\beta=\delta+\frac{g}{2}\left(i+\alpha_{H}\right)+i D
$$

Here, $\delta$ is the background wave number measured relative to the Bragg resonance and $\alpha_{H}$ denotes the linewidth enhancement factor. The peak gain $g$ is a function of the carrier density $N$,

$$
g=\frac{g^{\prime}\left(N-N_{t r}\right)}{1+\varepsilon S}, S=\left|E^{+}\right|^{2}+\left|E^{-}\right|^{2}
$$

with $g^{\prime}$ as differential gain, including the transverse confinement factor, $N_{t r}$ as transparency concentration, and $\varepsilon$ accounting for nonlinear gain saturation. The optical field is normalized so that $S$ represents the local photon density. Dispersive contributions are taken into consideration by the operator $D$ reading as

$$
D E^{ \pm}=\frac{\bar{g}}{2}\left(p^{ \pm}-E^{ \pm}\right)
$$

For the polarization $p^{ \pm}$, a single-oscillator model is used:

$$
\frac{\partial}{\partial t} p^{ \pm}=\bar{\gamma}\left(E^{ \pm}-p^{ \pm}\right)+i \omega p^{ \pm}
$$

where $\omega$ is the resonance frequency taken relative to the central frequency $2 \pi c / \lambda_{0}, g-\bar{g}$ the off-resonance gain, and $\bar{\gamma}$ measures the gain bandwidth.

The carrier densities $N(t, z)$ in the DFB and amplifier section are solutions of the rate equation

$$
\frac{\partial}{\partial t} N=J-R(N)-2 v_{g} \operatorname{Im}\left(E^{*} \beta E\right)
$$

The terms on the right-hand side are the injection rate $J$, the spontaneous recombination $R(N)=A N+B N^{2}+C N^{2}$, as well as the stimulated emission. 


\section{Appendix C}

\section{Device parameter in simulation}




\begin{tabular}{|c|l|c|c|c|l|}
\hline & explanation & \multicolumn{3}{|c|}{ values } & unit \\
\hline$\kappa$ & index coupling coeff. & 130 & 0 & 0 & $\mathrm{~cm}^{-1}$ \\
$l$ & section length & 200 & 350 & 250 & $\mu m$ \\
$\sigma$ & cross-section of AZ & 0.45 & & 0.45 & $\mu \mathrm{m}^{2}$ \\
$g^{\prime}$ & effective diff. gain & 9 & & 9 & $10^{-17} \mathrm{~cm}^{2}$ \\
$\alpha_{H}$ & Henry factor & -5 & & -5 & \\
$\alpha$ & internal absorption & 25 & {$[20,40]$} & 25 & $\mathrm{~cm}^{-1}$ \\
$\delta$ & static detuning & 402.7 & & {$[0,160]$} & $\mathrm{cm}^{-1}$ \\
$I$ & current injection & 70 & & {$[0,100]$} & $\mathrm{mA}^{\mathrm{A}}$ \\
$N_{t r}$ & transp. carrier density & 1 & & 1 & $10^{18} \mathrm{~cm}^{-3}$ \\
$A$ & recombination coeff. & 0.3 & & 0.3 & $10^{9} \mathrm{~s}^{-1}$ \\
$B$ & recombination coeff. & 1 & & 1 & $10^{-10} \mathrm{~cm}^{3} \mathrm{~s}^{-1}$ \\
$C$ & recombination coeff. & 1 & & 1 & $10^{-28} \mathrm{~cm}^{6} \mathrm{~s}^{-1}$ \\
$\varepsilon$ & nonl. gain comp. & 3 & & 3 & $10^{-18} \mathrm{~cm}^{3}$ \\
$U_{F}^{\prime}$ & diff. Fermi level sep. & 1 & & 1 & $10^{-19} \mathrm{~V} / \mathrm{cm}^{3}$ \\
$R_{s}$ & series resistivity & 5 & & 5 & $\Omega$ \\
$\bar{g}$ & Lorentzian height & 200 & 0 & 200 & $\mathrm{~cm}$ \\
$\bar{\gamma}$ & Lorentzian half width & 23.84 & & 23.84 & $\mathrm{rad} / \mathrm{ps}^{-1}$ \\
$\bar{\omega}$ & Lorentzian central f. & 2.384 & & 2.384 & $\mathrm{rad} / \mathrm{ps}^{2}$ \\
$\lambda_{0}$ & central wavelength & 1540 & & & $\mathrm{~nm}$ \\
$R$ & power reflectivity & & & 0.3 & \\
$v_{g}$ & group velocity & & $c / 3.8$ & & \\
\hline
\end{tabular}

Table C.1: Parameter values used for the DFB, phase tuning (P), and amplifier (A) sections. Deviations are noted in the text. 


\section{Bibliography}

[AdSCL04] Avila, J. F. M.; de S. Cavalcante, H. L. D.; ; Leite, J. R. R.: Experimental deterministic coherence resonance. In: PHYSICAL REVIEW LETTERS, volume 93(14):p. 144101, October 2004.

[Arn98] Arnold, L: Random Dynamical Systems. Springer, Springer, 5th edition, 1998.

[ASY06] Alligood, K.T.; Sander, E.; Yorke, J. A.: Chaotic attractors in crisis. In: PHYSICAL REVIEW LETTERS, volume 96(24):p. 244103, June 2006.

[Bau05] Bauer, Stefan: Nonlinear dynamics of semiconductor lasers with active optical feedback. Ph.D. thesis, MathematischNaturwissenschaftliche Fakultät der Humboldt-Universität zu Berlin, FB 3, 2005. Erschienen in der DISKI-Reihe mit der Nummer 216.

$\left[\mathrm{BBB}^{+} 03\right]$ Bauer, S.; Brox, O.; Biletzke, M.; Kreissl, J.; Radziunas, M.; B.Sartorius; Wünsche, H.-J.: Speed Potential of Active Feedback Lasers. In: Lasers and Electro-Optics Europe, CLEO/Europe., Informatik Aktuell, p. CC1M. Europhysics Conference Abstracts, Munich, 2003.

[BBK $\left.{ }^{+} 02\right]$ Bauer, S.; Brox, O.; Kreissl, J.; Sahin, G.; Sartorius, B.: Optical microwave source. In: ELECTRONICS LETTERS, volume 38(7):pp. 334-335, March 2002.

$\left[\mathrm{BBK}^{+} 04\right]$ Bauer, S.; Brox, O.; Kreissl, J.; Sartorius, B.; Radziunas, M.; Sieber, J.; Wünsche, H.-J.; Henneberger, F.: Nonlinear dynamics of semiconductor lasers with active optical feedback. In: PHYSICAL REVIEW E, volume 69(1):p. 016206, January 2004. 
[Bet03] Betke, Alexander: Erregbarkeit in MehsektionsHalbleiterlasern. Master's thesis, MathematischNaturwissenschaftliche Fakultät der Humboldt-Universität zu Berlin, 2003.

[Bro05] Brox, Olaf: DFB-Laser mit integriert optischer Rückkopplung für die optische Signalverarbeitung. Ph.D. thesis, Technische Universität Berlin, FB 3, 2005. Erschienen in der DISKI-Reihe mit der Nummer 216.

[BSdA90] Bennet, B. R.; Soref, R. A.; del Alamo, J. A.: Carrier-induced change in refractive index of inp, gaas, and ingaasp. In: IEEE JOURNAL OF SELECTED TOPICS IN QUANTUM ELECTRONICS, volume 26(1):pp. 113-122, January 1990.

[BSV81] Benzi, R.; Sutera, A.; Vulpiani, A.: The mechanism of stochastic resonance. In: JOURNAL OF PHYSICS A-MATHEMATICAL AND GENERAL, volume 14(11):pp. L453-L457, November 1981.

[BWSM97] Bandelow, U.; Wünsche, H.-J.; Sartorius, B.; Möhrle, M.: Dispersive self-q-switching in dfb lasers - theory versus experiment. In: IEEE JOURNAL OF SELECTED TOPICS IN QUANTUM ELECTRONICS, volume 3(2):pp. 270-278, April 1997.

[CB99] Clother, D. R.; Brindley, J.: Excitability of an age-structured plankton ecosystem. In: JOURNAL OF MATHEMATICAL BIOLOGY, volume 39(5):pp. 377-420, November 1999.

[CH93] Cross, M.C.; Hohenberg, D.C.: Pattern formation outside of equilibrium. In: REVIEWS OF MODERN PHYSICS, volume 65(3):pp. 851-1112, July 1993.

[CMT01] Calvo, O.; Mirasso, C.R.; Toral, R.: Coherence resonance in chaotic electronic circuits. In: ELECTRONICS LETTERS, volume 37(17):pp. 1062-1063, August 2001.

[CWP98] Carroll, John E.; Whiteaway, J.E.A.; Plumb, R.G.S.: Distributed Feedback Semiconductor Lasers. IEE Publishing, Springer, 1st edition, 1998.

[DRC+89] Ditto, W. L.; Rauseo, S.; Cawley, R.; Grebogi, C.; Hsu, G.H.; Kostelich, E.; Ott, E.; Savage, H. T.; Segnan, R.; Spano, 
M. L.; Yorke, J. A.: Experimental observation of crisis-induced intermittency and its critical exponent. In: ELECTRONICS LETTERS, volume 63(9):pp. 923-926, August 1989.

[DWK99] Dinklage, A.; Wilke, C.; Klinger, T.: Spatio-temporal response of stochastic resonance in an excitable discharge plasma. In: PHYSICS OF PLASMAS, volume 6(8):pp. 29682971, August 1999.

[EHRSG86] Ebeling, W.; Herzel, H.; Richert, W.; Schimansky-Geier, L.: Influence of noise on duffing-van der pol oscillators. In: ZEITSCHRIFT FUR ANGEWANDTE MATHEMATIK UND MECHANIK, volume 66(3):pp. 141-146, March 1986.

[EO93] Etrich, C.; Otsuka, K.: Laser rate equations with phase sensitive interactions. In: IEEE JOURNAL OF QUANTUM ELECTRONICS, volume 29(3):pp. 836-843, March 1993.

[ERGK00] Erneux, T.; Rogister, F.; Gavrielides, A.; Kovanis, V.: Bifurcation to mixed external cavity mode solutions for semiconductor lasers subject to optical feedback. In: OPTICS COMMUNICATIONS, volume 183(5-6):pp. 467-477, September 2000 .

[FHME98] Fischer, I.; Heil, T.; Münkel, M.; Elsäßer, W.: On the mechanism of the LFF phenomenon in the coherence collapse of semiconductor lasers There is hope for Sisyphus! In: Physics and Simulation of Optoelectronic Devices VI, 3283, pp. 571579. SPIE-The International Society for Optical Engineering, 1998.

[FTL ${ }^{+}$96] Fischer, I.; Tartwijk, G. H. M.; Levine, A. M.; Elsäßer, W.; Göbel, E.; Lenstra, D.: Fast pulsing and chaotic itinerancy with a drift in the coherence collapse of semiconductor lasers. In: PHYSICAL REVIEW LETTERS, volume 76(2):pp. 220223, January 1996.

[GDNH93] Gang, H.; Ditzinger, T.; Ning, C.Z.; Haken, H.: Stochastic resonance without external periodic force. In: PHYSICAL REVIEW LETTERS, volume 71(6):pp. 807-810, August 1993.

[GGBT00] Giacomelli, Giovanni; Giudici, Massimo; Balle, Salvador; Tredicce, Jorge R.: Experimental evidence of coherence res- 
onance in an optical system. In: PHYSICAL REVIEW LETTERS, volume 84(15):pp. 3298-3301, apr 2000.

[GH83] Guckenheimer, J.; Holmes, P.: Nonlinear Oscillations, Dynamical Systems and Bifurcations of Vector Fields. Springer, New-York, 1st edition, 1983.

[Goo89] Goodman, M. R.: Study Notes in System Dynamics. Pegasus Communications, Pegasus, 1st edition, 1989.

[GORY87] Grebogi, C.; Ott, E.; Romeiras, F.; Yorke, J.A.: Critical exponents for crisis-induced intermittency. In: PHYSICAL REVIEW A, volume 36(11):pp. 5365-5380, December 1987.

[GOY82] Grebogi, C.; Ott, E.; Yorke, J. A.: Chaotic attractors in crisis. In: PHYSICAL REVIEW LETTERS, volume 48(22):pp. 1507-1510, September 1982.

[GOY86] Grebogi, C.; Ott, E.; Yorke, J. A.: Critical exponent of chaotic transients in nonlinear dynamical systems. In: PHYSICAL REVIEW LETTERS, volume 57(11):pp. 1284-1287, September 1986 .

[Hak75a] Haken, H.: Analogy between higher instabilities in fluids and lasers. In: PHYSICAL LETTERS A, volume 53(1):pp. 77-78, September 1975.

[Hak75b] Haken, H.: Cooperative phenomena in systems far from thermal equilibrium and in nonphysical systems. In: REVIEWS OF MODERN PHYSICS, volume 47(1):pp. 67-121, January 1975 .

[Hak83a] Haken, H.: Laser Theory. Springer, Berlin and New York, 3rd edition, 1983.

[Hak83b] Haken, H.: Synergetics. An introduction. Springer, Berlin, 3rd edition, 1983.

[HFE $\left.{ }^{+} 03\right]$ Heil, T.; Fischer, I.; Elsäßer, W.; Krauskopf, B.; Green, K.; Gavrielides, A.: Dynamics of semiconductor lasers subject to delayed optical feedback the short cavity regime. In: PHYSICAL REVIEW E, volume 67(6):p. 066214, June 2003. 
[HFEG01] Heil, T.; Fischer, I.; Elsäßer, W.; Gavrielides, A.: Dynamics of semiconductor lasers subject to delayed optical feedback the short cavity regime. In: PHYSICAL REVIEW LETTERS, volume 87(24):p. 243901, December 2001.

[HL67] Hempstead, R.D.; Lax, M.: Classical noise. vi. noise in selfsustained oscillators near threshold. In: PHYSICAL REVIEW, volume 161(2):pp. 350-366, February 1967.

[Hod47] Hodgkin, A.L.: The local electric changes associated with repetitive action in a non-medullated axon. In: JOURNAL OF PHYSIOLOG Y LONDON, volume 107(2):pp. 165-181, February 1947.

[HX99] Hou, Z.; Xin, H.: Noise-induced oscillation and stochastic resonance in an autonomous chemical reaction system. In: PHYSICAL REVIEW E, volume 60(6):pp. 6329-6332, December 1999.

[Izh00] Izhikevich, E.M.: Neural excitability, spiking and bursting. In: INTERNATIONAL JOURNAL OF BIFURCATION AND CHAOS, volume 10(10):pp. 1171-1266, June 2000.

[JC02] Jeon, G. S.; Choi, M. Y.: Autonomous stochastic resonance in fully frustrated josephson-junction ladders. In: PHYSICAL REVIEW B, volume 66(6):p. 064514, August 2002.

[JFT94] Jánosi, I. M.; Flepp, L.; Tél, T.: Exploring transient chaos in an nmr-laser experiment. In: PHYSICAL REVIEW LETTERS, volume 73(25):pp. 529-532, July 1994.

[KHSP03] Kissa, I. Z.; Hudson, J. L.; Santos, G. J. E.; Parmananda, P.: Experiments on coherence resonance: Noisy precursors to hopf bifurcations. In: PHYSICAL REVIEW E, volume 67(3):p. 035201(R), March 2003.

[KL00] Katsev, S.; L'Heureux, I.: Impact of environmental noise on oscillatory pattern formation in crystal growth: Plagioclase feldspar. In: PHYSICAL REVIEW E, volume 61(5):pp. 49724979, May 2000.

[KLGZ06] Khovanov, I. A.; L.Schimansky-Geier; Zaks, M.A.: Spectral analysis of noisy oscillators near hopf bifurcations. In: $A C T A$ 
PHYSICA POLONICA B, volume 37(5):pp. 1551-1560, May 2006.

[KP06] Kloeden, P. E.; Platen, E.: Numerical Solution of Stochastic Differential Equations. Springer, Berlin, 3rd edition, 2006.

[Kra40] Kramers, H. A.: Brownian motion in a field of force and the diffusion model of chemical reactions. In: PHYSICA, volume 7(7):pp. 284-304, July 1940.

[Kra00] Krauskopf, B.: Bifurcation analysis of laser systems. In: AIP Conference Proceedings Nonlinear Laser Dynamics: Concepts, Mathematics, Physics, and Applications International Spring School, 548, pp. 1-30. American Institute of Physics, 2000.

[KRWH03] Korneyev, N.; Radziunas, M.; Wünsche, H.-J.; Henneberger, F.: Bifurcations of a DFB Laser with Short Optical Feedback: Numerical Experiment. In: Physics and Simulation of Optoelectronic Devices XI, 4986, pp. 480-489. SPIE-The International Society for Optical Engineering, 2003.

[KS72] Kogelnik, H.; Shank, C. V.: Coupled-wave theory of distributed feedback lasers. In: JOURNAL OF APPLIED PHYSICS, volume 43(5):pp. 2327-2335, May 1972.

[KSS $\left.{ }^{+} 03\right]$ Krauskopf, B.; Schneider, K. R.; Sieber, J.; Wieczorek, S. W.; Wolfrum, M.: Excitability and self-pulsations near homoclinic bifurcations in semiconductor laser systems. In: OPTICS COMMUNICATIONS, volume 215(4-6):pp. 367-379, January 2003.

[LGONSG04] Linder, B.; Garcia-Ojalvo, J.; Neiman, A.; Schimansky-Geier, L.: Effects of noise in excitable systems. In: PHYSICS REPORTS-REVIEW SECTION OF PHYSICS LETTERS, volume 392(6):pp. 321-424, March 2004.

[LJ95] L.I; J.Liu: Experimental observation of stochastic resonancelike behavior of autonomous motion in weakly ionized $\mathrm{rf}$ magnetoplasmas. In: PHYSICAL REVIEW LETTERS, volume 74(16):pp. 3161-3164, April 1995.

[LK80] Lang, R.; Kobayashi, K.: External optical feedback effects on semiconductor injection laser properties. In: IEEE JOURNAL 
OF QUANTUM ELECTRONICS, volume 16(3):pp. 347-355, March 1980.

[LLBS03] Lai, Y.-C.; Liu, Z.; Billings, L.; Schwartz, I. B.: Noise-induced unstable dimension variability and transition to chaos in random dynamical systems. In: PHYSICAL REVIEW E, volume 67(2):p. 026210, February 2003.

[LNK98] Lee, S. G.; Neiman, A.; Kim, S.: Coherence resonance in a hodgkin-huxley neuron. In: PHYSICAL REVIEW E, volume 57(3):pp. 3292 - 3297, March 1998.

[LSG00] Lindner, B.; Schimansky-Geier, L.: Coherence and stochastic resonance in a two-state system. In: PHYSICAL REVIEW E, volume 61(6):pp. 6103-6110, June 2000.

[LSGL02] Lindner, B.; Schimansky-Geier, L.; Longtin, A.: Maximizing spike train coherence or incoherence in the leaky integrateand-fire model. In: PHYSICAL REVIEW E, volume 66(3):p. 031916, September 2002.

[MA98] Masoller, C.; Abraham, N. B.: Stability and modulation properties of a semiconductor laser with weak optical feedback from a distant reflector. In: QUANTUM AND SEMICLASSICAL OPTICS, volume 10(10):pp. 519-534, June 1998.

[MTM92] Mörk, J.; Tromborg, B.; Mark, J.: Chaos in semiconductor lasers with optical feedback: Theory and experiment. In: IEEE JOURNAL OF QUANTUM ELECTRONICS, volume 28(1):pp. 93-108, January 1992.

[Mur93] Murray, J. D.: Mathematical Biology. Springer, New York, 3rd edition, 1993.

[MWR88] McNamara, B.; Wiesenfeld, K.; Roy, R.: Observation of stochastic resonance in a ring laser. In: PHYSICAL REVIEW LETTERS, volume 60(25):pp. 2626-2629, June 1988.

[NLSGaM99] Neiman, A.; L. Schimansky-Geier and, A. Cornell-Bell; Moss, F.: Noise-enhanced phase synchronization in excitable media. In: PHYSICAL REVIEW LETTERS, volume 83(23):pp. 4896-4899, December 1999. 
[NP77] Nicolis, G.; Prigogine, I.: Self-Organization in NonEquilibrium Systems. Wiley, New York, 3rd edition, 1977.

[NSS97] Neiman, A.; Saparin, P. I.; Stone, L.: Coherence resonance at noisy precursors of bifurcations in nonlinear dynamical systems. In: PHYSICAL REVIEW E, volume 56(1):pp. 270-273, July 1997.

[Oht02] Ohtsubo, J.: Introduction to the feature section on optical chaos and applications to cryptography. In: IEEE JOURNAL OF QUANTUM ELECTRONICS, volume 38(9):pp. 11411154, September 2002.

[PDEPH88] Papoff, F.; Dangoisse, D.; E. Poite-Hanoteau, P. Glorieux: Chaotic transients in a co2 laser with modulated parameters: critical slowing-down and crisis-induced intermittency. In: OPTICS COMMUNICATIONS, volume 67(5):pp. 358362, August 1988.

[Pet95] Petermann, K.: External optical feedback phenomena in semiconductor lasers. In: IEEE JOURNAL OF SELECTED TOPICS IN QUANTUM ELECTRONICS, volume 1(2):pp. 480489, June 1995.

[PHYS99] Postnov, D.E.; Han, S. K.; Yim, T. G.; Sosnovtseva, O.V.: Experimental observation of coherence resonance in cascaded excitable systems. In: PHYSICAL REVIEW E, volume 59(4):pp. R3791- R3794, April 1999.

[PK97] Pikovsky, A.; Kurths, J.: Coherence resonance in a noisedriven excitable system. In: PHYSICAL REVIEW LETTERS, volume 78(5):pp. 775-778, February 1997.

[POC99] Pradines, J.R.; Osipov, G.V.; Collins, J.J.: Coherence resonance in excitable and oscillatory systems: The essential role of slow and fast dynamics. In: PHYSICAL REVIEW E, volume 60(6):pp. 6407-6410, December 1999.

[PTMC01] Palenzuela, C.; Toral, R.; Mirasso, C.R.; Calvo, O.: Coherence resonance in chaotic systems. In: EUROPHYSICS LETTERS, volume 56(3):pp. 347-353, November 2001. 
[Rad06] Radziuna, M.: Numerical bifurcation analysis of the traveling wave model of multisection semiconductor lasers. In: PHYSICAL REVIEW E, volume 213(1):pp. 98-112, January 2006.

[Ris89] Risken, H.: Fokker-Planck equation. Methods of Solution and Applications. Springer, Berlin, 1st edition, 1989.

[RTAW01] Rozenfeld, A. F.; Tessone, C. J.; Albano, E.; Wio, H. S.: Universal critical behavior of noisy coupled oscillators. In: PHYSICAL LETTERS A, volume 280(1-2):pp. 45-52, February 2001.

[RWS $\left.{ }^{+} 00\right]$ Radziunas, M.; Wünsche, H.-J.; Sartorius, B.; Brox, O.; Hoffmann, D.; Schneider, K.; Marcenac, D.: Self-pulsating $\mathrm{dfb}$ lasers with an integrated phase tuning section. In: IEEE JOURNAL OF QUANTUM ELECTRONICS, volume 36(9):pp. 1026-1034, September 2000.

[San94] Sano, T.: Antimode dynamics and chaotic itinerancy in the coherence collapse of semiconductor lasers with optical feedback. In: PHYSICAL REVIEW A, volume 50(3):pp. 27192726, September 1994.

[SDG $\left.{ }^{+} 91\right]$ Sommerer, J. C.; Ditto, W. L.; Grebogi, C.; Ott, E.; Spano, M. L.: Experimental confirmation of the theory for critical exponents of crises. In: PHYSICAL LETTERS A, volume 153(2-3):pp. 105-109, February 1991.

[SMRE95] Sartorius, B.; Mohrle, M.; Reichenbacher, S.; Ebert, W.: Controllable self-pulsations in multi-section dfb lasers with an integrated phase-tuning section. In: IEEE PHOTONICS TECHNOLOGY LETTERS, volume 7(11):pp. 1261-1263, November 1995.

[SPM04] Sciamanna, M.; P.Megret; M.Blondel: Hopf bifurcation cascade in small-a laser diodes subject to optical feedback. In: PHYSICAL REVIEW E, volume 69(4):p. 046209, April 2004.

[Str01] Strogatz, S. H.: Nonlinear Dynamics and Chaos. Perseus Books Publishing, Cambridge, 1st edition, 2001.

[TLO94] Tromborg, B.; Lassen, H. E.; Olesen, H.: Travelling wave analysis of semiconductor lasers. In: IEEE JOURNAL OF QUANTUM ELECTRONICS, volume 30(4):pp. 939-956, April 1994. 
[TP94] Tager, A. A.; Petermann, K.: High-frequency oscillations and self-mode locking in short external-cavity laser diodes. In: IEEE JOURNAL OF QUANTUM ELECTRONICS, volume 30(7):pp. 1553-1561, July 1994.

[TRJ04] T. Risler, J. Prost; Jülicher, F.: Universal critical behavior of noisy coupled oscillators. In: PHYSICAL REVIEW LETTERS, volume 93(17):p. 175702, October 2004.

[TWS $\left.{ }^{+} 00\right]$ Tronciu, V.Z.; Wünsche, H.-J.; Sieber, J.; Schneider, K.; Henneberger, F.: Dynamics of single mode semiconductor lasers with passive dispersive reflectors. In: OPTICS COMMUNICATIONS, volume 182(1-3):p. 221228, August 2000.

$\left[\mathrm{UBB}^{+} 04\right]$ Ushakov, O.; Bauer, S.; Brox, O.; Wünsche, H.-J.; Henneberger, F.: Self- organization in semiconductor lasers with ultrashort optical feedback. In: PHYSICAL REVIEW LETTERS, volume 92(4):p. 043902, January 2004.

[UBOBH04] Ushakov, O.; Bauer, S.; O. Brox, H-J. Wünsche; Henneberger, F.: Dynamics of lasers with ultra-short optical feedback. In: Physics and Simulation of Optoelectronic Devices XII, 5349, pp. 348-357. SPIE-The International Society for Optical Engineering, 2004.

[UKR $\left.{ }^{+} 06\right]$ Ushakov, O. V.; Korneyev, N.; Radziunas, M.; Wünsche, H.J.; Henneberger, F.: Excitability of chaotic transients in a semiconductor laser. In: submitted to EURO PHYSICS LETTERS, 2006.

[UWFH $\left.{ }^{+} 05\right]$ Ushakov, O.V.; Wünsche, H.-J.; F. Henneberger, I. A. Khovanov; Schimansky-Geier, L.; Zaks, M. A.: Coherence resonance near a hopf bifurcation. In: PHYSICAL REVIEW LETTERS, volume 95(12):p. 123903, September 2005.

[vTA86] van Tartwijk, G. H. M.; Agrawal, G. P.: Regimes of feedback effects in 1.5 micrometer distibuted feedback lasers. In: JOURNAL OF LIGHTWAVE TECHNOLOGY, volume 4(11):pp. 1655-1661, November 1986.

[vTA98] van Tartwijk, G. H. M.; Agrawal, G. P.: Laser instabilites: a modern perspective. In: PROGRESS IN QUANTUM ELECTRONICS, volume 22(2):pp. 43-122, February 1998. 
[WBRH02] Wünsche, H-J.; Brox, O.; Radziunas, M.; Henneberger, F.: Excitability of a semiconductor laser by a two-mode homoclinic bifurcation. In: PHYSICAL REVIEW LETTERS, volume 88(2):p. 023901, January 2002.

[WBWR96] Wenzel, H.; Bandelow, U.; Wünsche, H.-J.; Rehberg, J.: Mechanisms of fast self pulsations in two-section dfb lasers. In: IEEE JOURNAL OF QUANTUM ELECTRONICS, volume 32(1):pp. 69-79, January 1996.

[Wie85] Wiesenfeld, K.: Noisy precursor of nonlinear instabilities. In: JOURNAL OF STATISTICAL PHYSICS, volume 38(5-6):pp. 1071-1097, June 1985.

[WKL99] Wieczorek, S.; Krauskopf, B.; Lenstra, D.: Dynamics of single mode semiconductor lasers with passive dispersive reflectors. In: OPTICS COMMUNICATIONS, volume 172(1-6):pp. 279295, December 1999.

[WKL01] Wieczorek, S.; Krauskopf, B.; Lenstra, D.: Unnested islands of period doublings in an injected semiconductor laser. In: PHYSICAL REVIEW E, volume 64(5):p. 056204, November 2001.

[WSSV85] Wolf, A.; Swift, J.B.; Swinney, H.L.; Vastano, J.A.: Determining lyapunov exponents from a time series. In: PHYSICA $D$, volume 16(3):pp. 285-317, July 1985.

[WT02] Wolfrum, M.; Turaev, D.: Instabilities of lasers with moderately delayed optical feedback. In: OPTICS COMMUNICATIONS, volume 212(1-3):pp. 127-138, October 2002.

[WV91] Weiss, C. O.; Vilaseca, R.: Dynamics of Lasers. Wiley, Weinheim, 1st edition, 1991.

$\left[Z_{K A}^{+} 03\right]$ Zhou, C. S.; Kurths, J.; Allaria, E.; Boccaletti, S.; Meucci, R.; Arecchi, F. T.: onstructive effects of noise in homoclinic chaotic systems. In: PHYSICAL REVIEW E, volume 67(6):p. 066220, June 2003.

[ZZ70] Zaikin, A.; Zhabotinsky, A.: Concentration wave propation in 2-dimensional liquid-phase self-oscillator system. In: $N A$ TURE, volume 225(5232):p. 535, January 1970. 


\section{Abbreviation}

\begin{tabular}{ll} 
Abbreviation & Explanation \\
\hline AFL & active feedback laser \\
AR & anti-reflection \\
ASE & amplified spontaneous emission \\
BC & boundary crisis \\
CA & chaotic attractor \\
CC & coherence collapse \\
CR & coherence resonance \\
CS & chaotic saddles \\
CT & chaotic transients \\
cw & continuous wave \\
dc & direct current \\
DFB & distributed feedback \\
DQS & dispersive Q-switching \\
EC(M) & external cavity (mode) \\
ESA & electrical spectrum analyzer \\
FP & Fabry-Perot \\
FWHM & full-width at half maximum \\
LFF & low frequency fluctuations \\
LK & Lang-Kobayashi \\
MB & mode beating \\
OSA & optical spectrum analyzer \\
PD & period doubling \\
PDF & probability density function \\
PFL & passive feedback laser \\
rf & radio frequency \\
RO & relaxation oscillation \\
SL & semiconductor laser \\
SN & saddle-node \\
SNR & signal-to-noise ratio \\
SP & self-pulsation \\
SR & stochastic resonance \\
TWE & traveling-wave equation \\
URO & \\
&
\end{tabular}




\section{Acknowledgments}

First of all I would like to express my gratitude to Prof. Fritz Henneberger for having made it possible for me to make my Ph.D. in his scientific group. Especially I am indebted to Dr. Hans-Jurgen Wunsche who was my direct supervisor during my Ph.D. His careful and kind supervision as well as the fascinating ideas filled me all these years and contributed a great deal to my world view as well as to my scientific education. Further I would like to acknowledge Dr. Bernd Sartorius, Dr. Stefan Bauer, Dr. Olaf Brox and Fraunhofer-HHI Berlin, for providing the laser devices. As well I express my appreciation to Dr. Ingo Dudeck for the technical support in the lab. I would also like to acknowledge all my colleagues: Dr. Joachim Puls, Dipl. Phys. Alexander Betke, Dipl. Phys. Sylvia Schikora, and Martin Spreemann for creating a wonderful working atmosphere and helping me to figure out a number of small things which occur in everyday life. And last but not least I would like to thank my family for the kind support. 


\title{
Lebenslauf
}

\author{
Name: OlegUshakov \\ 09.1995-02.2001 Studium an der Lomonosov Moscow State University \\ in der Fachrichtung Physik \\ 06.2001-06.2002 Wissenschaftliche Mitarbeiterin an \\ der Stutgart Universiät, \\ Lehrstuhl Prof. M. Dressel, \\ 1. Physikalisches Institut \\ 06.2002 \\ Wissenschaftliche Mitarbeiterin an \\ der Humboldt-Universität zu Berlin, \\ Lehrstuhl Prof. F. Henneberger, \\ Institut für Physik
}




\section{Selbständigkeitserklärung}

Hiermit erkläre ich, die vorliegende Arbeit selbständig ohne fremde Hilfe verfaßt und nur die angegebene Literatur und Hilfsmittel verwendet zu haben. 